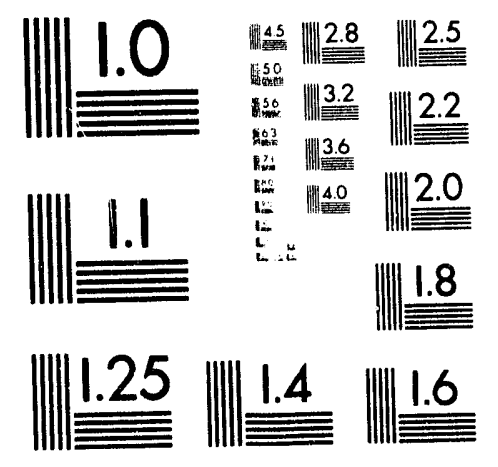



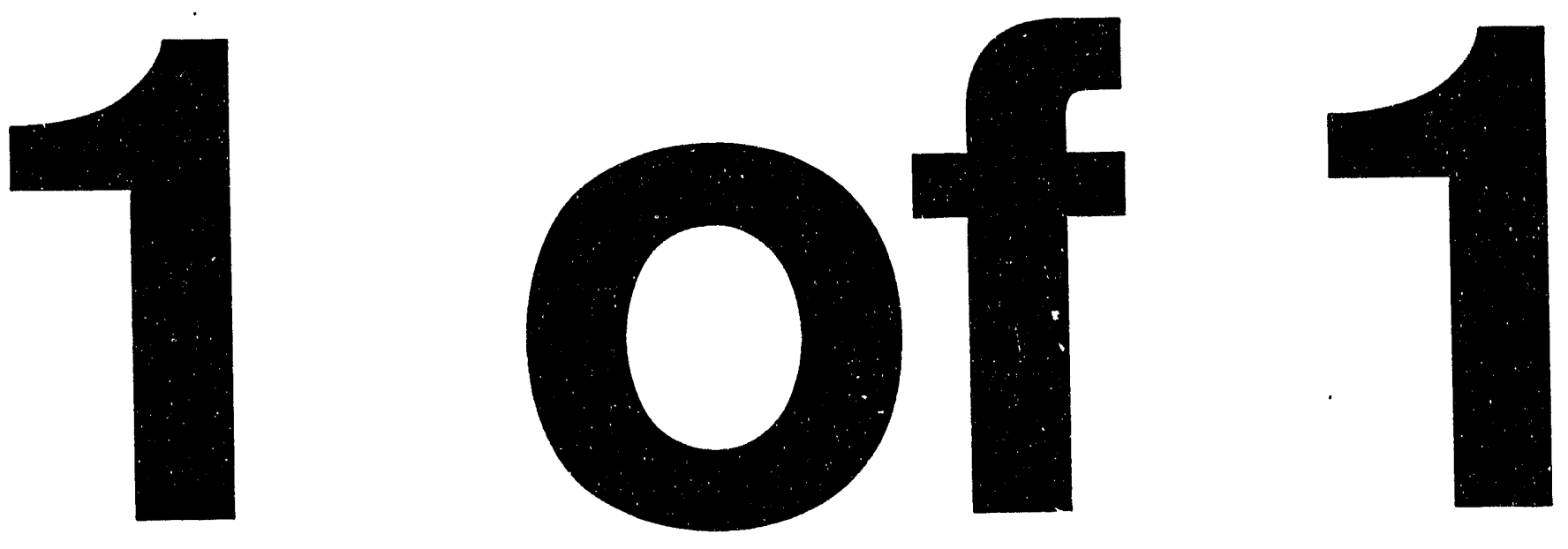


\title{
STOCHASTIC DIFFERENTIAL EQUATIONS AND NUMERICAL SIMULATION FOR PEDESTRIANS
}

\author{
John C. Garrison
}

Physical Sciences

V-Division

July 27,1993

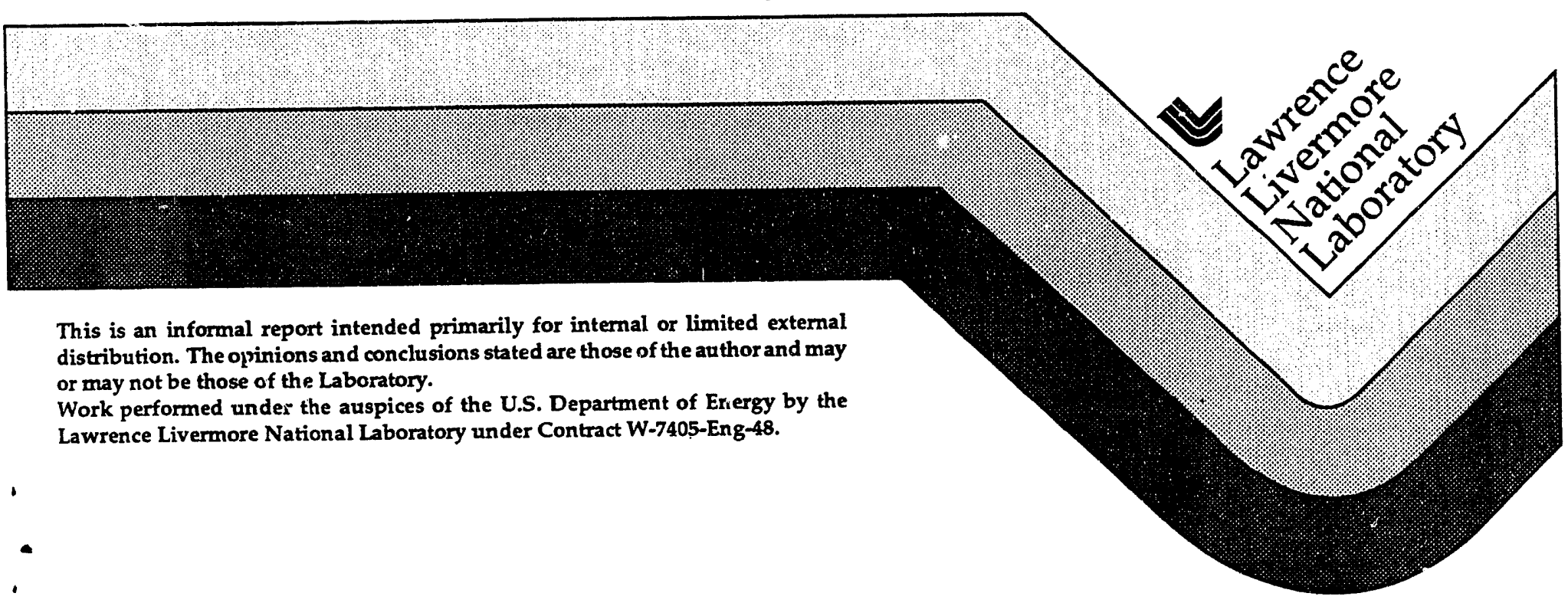

This is an informal report intended primarily for internal or limited external distribution. The oppinions and conclusions stated are those of the author and may or may not be those of the Laboratory.

Work performed under the auspices of the U.S. Department of Evergy by the Lawrence Livermore National Laboratory under Contract W-7405-Eng-48. 


\section{DISCLAIMER}

This document was prepared as an account of work sponsored by an agency of the United States Government. Neither the United States Government nor the University of California nor any of their employees, makes any warranty, express or implied, or assumes any legal liability or responsibility for the accuracy, completeness, or usefulness of any information, apparatus, product, or process disclosed, or represents that its use would not infringe privately owned rights. Reference herein to any specific commercial products, process, or service by trade name, trademark, manufacturer, or otherwise, foes not necessarily constitute or imply its endorsement, recommendation, or favoring by the United States Government or the University of California. The views and opinions of authors expressed herein do not necessarily state or reflect those of the United States Government or the University of California, and shall not be used for advertising or product endorsement purposes.

This report has been reproduced directly from the best available copy.

A vailable to DOE and DOE contractors from the Office of Scientific and Technical Information P.O. Box 62, Oak Ridge, TN 37831

Prices available from (615) 576-8401, FTS 626-8401

A vailable to the public from the

National Technical Information Service

US. Department of Commerce

5285 Port Royal Rd.,

Springfield, VA 22161 


\title{
STOCHASTIC DIFFERENTIAL EQUATIONS AND NUMERICAL SIMULATION FOR PEDESTRIANS
}

\author{
John C. Garrison
}

\begin{abstract}
The mathematical foundation of the Ito interpretation of stochastic ordinary and partial differential equations is briefly explained. This provides the basis for a review of simple difference approximations to stochastic differential equations. An example arising in the theory of optical switching is discussed.
\end{abstract}

\section{MASTER}

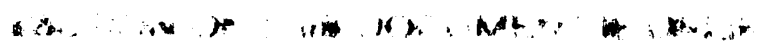




\section{Introduction}

Stochastic differential equations appear in many areas of science and engineering, ranging from morlels of turbulence, through thermal noise in circuits, to quantum noise in electronic and optical devices. This ubiquity means that those of us who are not experts in stochastic processes are nonetheless required to deal with this kind of mathematics. There are excellent monographs and papers on the mathematical foundations of the subject [1-6] and others expiaining the art of numericai simulation, [7-11] but the mathematical works generally ignore numerical methods, and the explanations of simulation techniques generally assume complete knowledge of the mathematical foundations. It therefore seems useful to combine a brief review of the mathematics with a description of simulation techniques.

The general field of stochastic processes is far too large to discuss in a short review; therefore, I will restrict myself to stochastic differential equations and simple methods for their numerical simulation. In discussing this subject it is necessary to make a choice between the competing approaches associated with the names of Stratonovich and Ito. This argument resembles a religious schism, so any choice will be objectionable to some of the cognoscenti. However, from the point of view of a practical user it would appear that the Ito calculus is easier to understand and to apply. I will therefore adopt the Ito approach and refer the interested reader to the literature for the fine points of the debate [6],[1]. In Sec. 2, the Ito calculus is explained by the canonical example of the Langevin equation. Finite difference schemes are reviewed in Sec. 3., and Sec. 4 explains numerical simulation techniques for generating the fundamental Wiener process and linear functionals of it. The extension of these ideas to deal with stochastic partial differential equations is outlined in Sec. 5., and in Sec. 6 an example arising in the theory of all-optical switching will be used to illustrate the derivation of stochastic equations for a real life problem.

\section{Ito Calculus}

The subject of stochastic differential equations began with the study of Brownian motion $[12,13]$ which led to the Langevin equation [14]. In modern notation the generic Langevin equation is

$$
\frac{d u}{d t}=a(u, t)+b(u, t) \eta(t)
$$

where the functions $a$ and $b$ are called the drift and diffusion terms respectively, and $\eta(t)$ represents random environmental forces. Typically it is assumed that $\eta(t)$ satisfies

$$
\langle\eta(t)\rangle=0
$$




$$
\left\langle\eta(t) \eta\left(t^{\prime}\right)\right\rangle=\delta\left(t-t^{\prime}\right)
$$

where $\langle\ldots\rangle$ represents an appropriate average. Physically, the apparent randomness in $\eta(t)$ arises from ignorance about the detailed time evolutinn of the environment (usually referred to as a "reservoir", or as a "heat bath" for situations of thermal equilibrium), and the delta function in (2.2.2) is an idealization of the assumption that correlation times of reservoir variables are much shorter than any characteristic time for the system property u. For time-stationary processes, the correlation function $\left\langle\eta(t) \eta\left(t^{\prime}\right)\right\rangle$ is a function of the time difference $\left(t-t^{\prime}\right)$ only, and the Fourier transform of the correlation function represents the power spectrum of the noise. Thus (2.2.2) shows that the power spectrum is independent of $\omega$; for this reason fluctuations obeying (2.2) are called white noise.

Mathematically, (2.1) and (2.2) are simply meaningless as they stand. This lack of rigor is not merely an offense against mathematical esthetics, it is a significant practical problem. The point is that it is impossible to devise a reliable and meaningful numerica? scheme in the absence of a proper analytical foundation. The first step in legitimizing the Langevin equation is to define what is meant by a "random" variable. The standard procedure is to introduce a probability space $(\Omega, \rho)$, where $\Omega$ is the space of possible values of the variable and $\rho$ is a nonnegative density function defined on $\Omega$ which satisfies

$$
\int_{\Omega} d \zeta \rho(\zeta)=1
$$

A random variable, e.g., $\eta(t)$ is then interpreted as a function $\eta(t ; \zeta)$ and averages are defined by

$$
\langle\eta(t)\rangle=\int_{\Omega} d \zeta \rho(\zeta) n(t ; \zeta)
$$

Once this point is understood, explicit reference to the probability space $(\Omega, \rho)$ is usually unnecessary. The next difficulty is that the infinitely rapid and irregular temporal fluctuations described by (2.2.2) are inconsistent with the differentiability of $u(t)$. Ito and Stratonovich both treat this problem by replacing the differential equation (2.1) by the corresponding integral equation

$$
u(t)=u\left(t_{0}\right)+\int_{t_{0}}^{t} d t^{\prime} a\left(u\left(t^{\prime}\right), t^{\prime}\right)+\int_{t_{0}}^{t} b\left(u\left(t^{\prime}\right), t^{\prime}\right) d W\left(t^{\prime}\right)
$$

where $W(t)$ is related to $\eta(t)$ by 


$$
W(t)=\int_{t_{0}}^{t} \eta\left(t^{\prime}\right) d t^{\prime}
$$

The conditions in (2.2) together with (2.6) yield

$$
\begin{aligned}
& \langle W(t)\rangle=0, \\
& \left\langle\Delta W\left(t^{2}\right)^{2}\right\rangle=\Delta t, \\
& \left\langle\Delta W(t) \Delta W\left(t^{\prime}\right)\right\rangle=0, \\
& \Delta W(t) \equiv W(t+\Delta t)-W(t),
\end{aligned}
$$

where the intervals $[t, t+\Delta t]$ and $\left[\mathfrak{t}^{\prime}, t^{\prime}+\dot{\Delta}^{\prime}\right]$ in (2.7.3) are nonoverlapping. At this point the poorly defined $\eta(t)$, and the singular condition (2.2.2) are discarded in favor of $W(t)$ and (2.7). A stochastic variable satisfying (2.7) is called a Wiener process; it is a Gaussian Markov process [15] with statistically independent increments $\Delta \mathrm{W}(\mathrm{t})$. The differences between Stratonovich and Ito come from different choices of definition for the stochastic integral in the last term of (2.5). Ito uses the properties of the Wiener process to define the stochastic integral by a kind of Riemann-Stieltjes procedure.[16] An approximation to the general stochastic integral

$$
S=\int_{t_{0}}^{t} d t^{\prime} G\left(t^{\prime}\right) d W\left(t^{\prime}\right)
$$

where $G(t)$ is an arbitrary (possibly stochastic) function, is defined by dividing the interval $\left[t_{0}, t\right]$ into $n$ subintervals, by means of a partition $t_{0} \leq t_{1} \leq \ldots \leq t_{n-1} \leq t$, and choosing $\tau_{i} \varepsilon\left[t_{i-1}, t_{i}\right]$ by $\tau_{i}=\alpha t_{i}+(1-\alpha) t_{i-1}$, with $0 \leq \alpha \leq 1$. The corresponding approximation to $S$ is

$$
\mathrm{S}_{\mathrm{n}}=\sum_{\mathrm{i}=1}^{\mathrm{n}} \mathrm{G}\left(\tau_{\mathrm{i}}\right) \Delta \mathrm{W}_{\mathrm{i}}
$$

where $\Delta \mathrm{W}_{\mathrm{i}}=\mathrm{W}\left(\mathrm{t}_{\mathrm{i}}\right)-\mathrm{W}\left(\mathrm{t}_{\mathrm{i}-1}\right)$. For ordinary integrals the value of $\alpha$ is irrelevant in the limit $\mathrm{n} \rightarrow \infty$, but simple examples $\left\{\right.$ e.g., $\mathrm{G}(\mathrm{t})=\mathrm{W}(\mathrm{t})$, which yields $\left\langle\mathrm{S}_{\mathrm{n}}\right\rangle=\alpha\left(\mathrm{t}-\mathrm{t}_{0}\right)$. $\}$ show that the limit of (2.9) depends explicitly on the value of $\alpha$. This means that a particular choice is required in order to define the integral. Ito chooses $\alpha=0$; i.e., the integrand $G(t)$ is evaluated 
at the initial point of the subinterval. This apparently innocuous choice has rather surprising consequences which are discussed below. The integral is defined by

$$
\int_{t_{0}}^{t} d t^{\prime} G\left(t^{\prime}\right) d W\left(t^{\prime}\right)=\underset{n s-\lim _{n \rightarrow \infty}}{ } \sum_{i=1}^{n} G\left(t_{i-1}\right) \Delta W_{i},
$$

where the mean-square limit is defined by

$$
X=\underset{n \rightarrow \infty}{\operatorname{ms}-\lim _{n}} X_{n} \text { if and only if } \lim _{n \rightarrow \infty}\left\langle\left(X_{n}-X\right)^{2}\right\rangle=0
$$

A random variable $u(t)$ is defined to be a solution of the Langevin equation (2.1), which is rewritten in the form

$$
d u=a(u, t) d t+b(u, t) d W(t)
$$

if $u(t)$ satisfies (2.5) with the Ito definition for the stochastic integral. The special case $\mathrm{b}(\mathrm{u})=\mathrm{b}$, independent of $\mathrm{u}$, is called additive noise and the general case is referred to as multiplicative noise. The form of the approximating sums on the right-hand-side of (2.10) clearly suggests the way in which numerical simulations should be approached, and one such formulation will be described in the following section. Before doing so, it will be useful to list a few of the immediate consequences of the Ito definition (2.10).

Many of the results to follow hold only for nonanticipating functions: A function $G(t)$ is nonanticipating (with respect to the Wiener process $W(t)$ ) if $G(t)$ is statistically independent of $\left[W\left(t^{\prime}\right)-W(t)\right]$ for all $t^{\prime}>t$, i.e.,

$$
\left\langle G(t)\left[W\left(t^{\prime}\right)-W(t)\right]\right\rangle=0 \quad t^{\prime}>t
$$

In rnost applications $\mathrm{t}$ represents time so this definition imposes a notion of statistical causality. Examples of nonanticipating functions are:

$$
\begin{aligned}
& W(t), \\
& \int_{t_{0}}^{t} d t^{\prime} F\left[W\left(t^{\prime}\right)\right], \\
& \int_{t_{0}}^{t} d W\left(t^{\prime}\right) F\left[W\left(t^{\prime}\right)\right],
\end{aligned}
$$




$$
\begin{aligned}
& \int_{t_{0}}^{t} d t^{\prime} G\left(t^{\prime}\right), \text { for nonanticipating } G(t), \\
& \int_{t_{0}}^{t} d W\left(t^{\prime}\right) G\left(t^{\prime}\right), \text { for nonanticipating } G(t) \text {. }
\end{aligned}
$$

The particular choice of evaluation point made in Ito's definition has some peculiar consequences which violate the usual rules of calculus. The simplest example is

$$
\int_{t_{0}}^{t} W\left(t^{\prime}\right) d W\left(t^{\prime}\right)=\frac{1}{2}\left[W(t)^{2}-W\left(t_{0}\right)^{2}-\left(t-t_{0}\right)\right],
$$

which can be obtained by an exact calculation starting with (2.10). The extra term $\left(t-t_{0}\right)$ in (2.15) comes from the properties (2.7) of the Wiener process, which shows that $\Delta W(t) \approx \sqrt{\Delta t}$ (almost always). Thus terms of second order in $\Delta \mathrm{W}$ must be retained in the calculations. This feature is encapsulated in the formal rules

$$
\begin{aligned}
& d W(t)^{2}=d t \\
& d W(t)^{2+n}=0,
\end{aligned}
$$

which correspond to the precise statement

$$
\begin{aligned}
\int_{t_{0}}^{t} G\left(t^{\prime}\right)\left[d W\left(t^{\prime}\right)\right]^{2+n} & \equiv m_{n \rightarrow \infty}-\lim \sum_{i=1}^{n} G_{i-1}\left(\Delta W_{i}\right)^{2+n} \\
& =\delta_{n, 0} \int_{t_{0}}^{t} d t^{\prime} G\left(t^{\prime}\right)
\end{aligned}
$$

The proof of this identity depends on the fact that $G(t)$ is nonanticipating and that the $\Delta W_{i}$ are independent Gaussian random variables. Similarly

$$
\int_{t_{0}}^{t} d t^{\prime} G\left(t^{\prime}\right) d W\left(t^{\prime}\right) d t^{\prime}=\underset{n s-\lim }{\operatorname{ma}} \sum_{i=1}^{n} G_{i-1} \Delta W_{i} \Delta t_{i}=0
$$


The rule arising from these identities is that $\mathrm{dW}(\mathrm{t})$ is counted as an infinitesimal of order $1 / 2$ and that infinitesimals of order greater than 1 are to be dropped. An example of this rule is the calculation of differentials of powers of W. A formal expansion follc ved by use of $(2.16)$ gives

$$
\begin{aligned}
\mathrm{d}\left[\mathrm{W}(\mathrm{t})^{\mathrm{n}}\right] & =[\mathrm{W}(\mathrm{t})+\mathrm{dW}(\mathrm{t})]^{\mathrm{n}}-\left[\mathrm{W}(\mathrm{t})^{\mathrm{n}}\right] \\
& =\mathrm{nW}(\mathrm{t})^{\mathrm{n}-1} \mathrm{dW}(\mathrm{t})+\frac{\mathrm{n}(\mathrm{n}-1)}{2} \mathrm{~W}(\mathrm{t})^{\mathrm{n}-2} d t
\end{aligned}
$$

The additional term on the right hand side is one of the peculiarities of Ito calculus. This can be converted to an integral rule by solving $(2.18)$ for the $\mathrm{dW}$ term and integrating to obtain (after $n \rightarrow n+1)$

$$
\int_{t_{0}}^{t} d W\left(t^{\prime}\right) W\left(t^{\prime}\right)^{n}=\frac{1}{n+1}\left[W(t)^{n+1}-W\left(t_{0}\right)^{n+1}\right]-\frac{n}{2} \int_{t_{0}}^{t} d t^{\prime} W\left(t^{\prime}\right)^{n-1}
$$

An application of the same expansion and rules $\left(\mathrm{dW}^{2}=\mathrm{dt}, \mathrm{dW} d t=0, \mathrm{dt}^{2}=0\right.$ ) yields a useful result for arbitrary differentiable functions of the Wiener process

$$
\mathrm{df}[\mathrm{W}(\mathrm{t}), \mathrm{t}]=\left\{\frac{\partial \mathrm{f}}{\partial \mathrm{t}}+\frac{1}{2} \frac{\partial^{2} \mathrm{f}}{\partial \mathrm{W}^{2}}\right\} \mathrm{dt}+\frac{\partial \mathrm{f}}{\partial \mathrm{W}} \mathrm{dW}(\mathrm{t})
$$

Continuing in this vein leads to the Ito formula for a change of variables. To get this one asks for the stochastic differential equation obeyed by $f[u(t)]$, where $u(t)$ satisfies the Langevin equation (2.12) and $\mathrm{f}$ is a smooth function of its argument. Using the rules just given, the answer is

$$
\begin{aligned}
\operatorname{df}[u(t)] & =f[u(t)+d u(t)]-f[u(t)] \\
& =\partial_{u} f[u(t)] d u(t)+\frac{1}{2} \partial_{u}^{2} f[u(t)] d u(t)^{2} \\
& =\partial_{u} f[u(t)]\{a[u(t), t] d t+b[u(t), t] d W(t)\}+\frac{1}{2} b[u(t), t]^{2} \partial_{u}^{2} f[u(t)] d t,
\end{aligned}
$$

where $\partial_{u} f \equiv \partial f / \partial u$, etc.. The last term in the final line is a result of $\mathrm{dW}^{2}=\mathrm{dt}$ and would not appear in a standard chain rule calculation.

Useful properties of averages follow from the notion of nonanticipating functions. First note that the definition, (2.13), of a nonanticipating function $G(t)$ implies 


$$
\left\langle\int_{t_{0}}^{t} d W\left(t^{\prime}\right) G\left(t^{\prime}\right)\right\rangle=\underset{n s-\lim _{i=1}}{ } \sum_{i=1}^{n}\left\langle G_{i-1} \Delta W_{i}\right\rangle=0 .
$$

This result does not hold for the Stratonovich definition of the stochastic integral. For nonanticipating continuous functions $\mathrm{G}$ and $\mathrm{H}$, one finds

$$
\left\langle\int_{t_{0}}^{t} d W\left(t^{\prime}\right) G\left(t^{\prime}\right) \int_{t_{0}}^{t} d W\left(t^{\prime}\right) H\left(t^{\prime}\right)\right\rangle=\int_{t_{0}}^{t} d t^{\prime}\left\langle G\left(t^{\prime}\right) H\left(t^{\prime}\right)\right\rangle
$$

which is the mathematically respectable version of (2.2).

Finally the rules of Ito calculus together with the interpretation of the average in terms of a probability density can be used to derive the Fokker Planck equation associated with the Langevin equation.[2] To this end take the average of (2.21) to get

$$
\begin{aligned}
\langle d f[u]\rangle & =\left\langle\partial_{u} f[u(t)] a[u, t]\right\rangle d t+\left\langle\partial_{u} f b[u, t] d W(t)\right\rangle+\frac{1}{2}\left\langle b[u, t]^{2} \partial_{u}^{2} f[u]\right\rangle d t \\
& =\left\langle\partial_{u} f[u(t)] a[u, t]\right\rangle d t+\frac{1}{2}\left\langle b[u, t]^{2} \partial_{u}^{2} f[u]\right\rangle d t
\end{aligned}
$$

where the second line follows from the fact that $u(t)$ and functions of $u(t)$ are nonanticipating, so that the second term on the right hand side of the first line vanishes. The underlying probability space density $\rho$ can be used to define a conditional probability $p\left(u, t \mid u_{0}, t_{0}\right)$ as the probability that $u(t)=u$ given $u\left(t_{0}\right)=u_{0}$. Then averages can be expressed as

$$
\langle f[u(t)]\rangle=\int d u f[u] p\left(u, t \mid u_{0}, t_{0}\right)
$$

etc., and (2.24) can be rewritten as

$$
\int d u f[u] \partial_{t} p\left(u, t \mid u_{0}, t_{0}\right)=\int d u\left\{a(u, t) \partial_{u} f[u]+\frac{1}{2} b(u, t) \partial_{u}^{2} f(u]\right\} p\left(u, t \mid u_{0}, t_{0}\right) .
$$

The final step is to integrate by parts with respect to $u$ on the right hand side and assume that the surface terms can be dropped. Since the function $f(u)$ is arbitrary, this yields the FokkerPlanck equation for the probability density $\mathrm{p}\left(\mathrm{u}, \mathrm{t} \mid \mathrm{u}_{0}, \mathrm{t}_{0}\right)$ :

$$
\partial_{\mathrm{t}} p\left(\mathrm{u}, \mathrm{t} \mid \mathrm{u}_{0}, \mathrm{t}_{0}\right)=-\partial_{\mathrm{u}}\left\{\mathrm{a}(\mathrm{u}, \mathrm{t}) \mathrm{p}\left(\mathrm{u}, \mathrm{t} \mid \mathrm{u}_{0}, \mathrm{t}_{0}\right)\right\}+\frac{1}{2} \partial_{\mathrm{u}}^{2}\left\{\mathrm{~b}(\mathrm{u}, \mathrm{t}) \mathrm{p}\left(\mathrm{u}, \mathrm{t} \mid \mathrm{u}_{0}, \mathrm{t}_{0}\right)\right\} .
$$


Thus (2.27) and the Langevin equation (2.12) are equivalent descriptions of the same process. For this reason the coefficients $a$ and $b$ in (2.12) are called the drift and diffusion terms respectively. The equivale ${ }_{1}$ ce of the Langevin and Fokker-Planck equations is of great practical importance, since it means that one can choose the description that is most convenient for the task at hand.

The Langevin equation for a single variable $u$ can be simply extended to systems of equations :

$$
d u^{\lambda}=a^{\lambda}(u, t) d t+b^{\lambda \mu}(u, t) d W_{\mu}(t)
$$

where $\lambda, \mu=1, \cdots, \mathrm{n}$ and the summation convention for repeated indices is in force. The $\mathrm{dW}_{\lambda}(\mathrm{t})$ are independent Wiener processes satisfying

$$
\begin{aligned}
& \left\langle\mathrm{W}_{\lambda}(\mathrm{t})\right\rangle=0 \\
& \left\langle\Delta \mathrm{W}_{\lambda}(\mathrm{t}) \Delta \mathrm{W}_{\mu}(\mathrm{t})\right\rangle=\delta_{\lambda \mu} \Delta \mathrm{t}, \\
& \left\langle\Delta \mathrm{W}_{\lambda}(\mathrm{t}) \Delta \mathrm{W}_{\mu}\left(\mathrm{t}^{\prime}\right)\right\rangle=0, .
\end{aligned}
$$

where $\Delta W_{\lambda}(t) \equiv W_{\lambda}(t+\Delta t)-W_{\lambda}(t)$, and the intervals $[t, t+\Delta t]$ and $\left[t^{\prime}, t^{\prime}+\Delta t^{\prime}\right]$ in (2.29.3) are nonoverlapping. The corresponding Fokker-Planck equation is

$$
\partial_{\mathrm{t}} \mathrm{p}\left(\mathbf{u}, \mathrm{t} \mid \mathbf{u}_{0}, \mathrm{t}_{0}\right)=-\partial_{\lambda}\left\{\mathrm{a}^{\lambda}(\mathbf{u}, \mathrm{t}) \mathrm{p}\left(\mathbf{u , t} \mid \mathbf{u}_{0}, \mathrm{t}_{0}\right)\right\}+\frac{1}{2} \partial_{\lambda} \partial_{\mu}\left\{\mathrm{b}^{\lambda \mu}(\mathbf{u}, \mathrm{t}) \mathrm{p}\left(\mathbf{u}, \mathrm{t} \mid \mathbf{u}_{0}, \mathrm{t}_{0}\right)\right\}
$$

where $\mathbf{u} \equiv\left(\mathbf{u}^{1}, \cdots, \mathrm{u}^{\mathrm{n}}\right)$, and $\partial_{\lambda} \equiv \partial / \partial \mathrm{u}^{\lambda}$.

\section{Finite difference schemes: ordinary differential equations}

In order to simplify the exposition and concentrate on the essential aspects, I will first discuss the autonomous scalar Langevin equation, i.e., the single-component equation (2.12) with coefficients $a(u)$ and $b(u)$ having no explicit time dependence, and then outline the extra difficulties that arise for vector Langevin equations, i.e., systems of two or more equations.

\section{3a Autonomous scalar Langevin equation}

The Ito form of the equation is

$$
d u=a(u) d t+b(u) d W(t)
$$


and the finite step method starts by applying $(2.5)$ to the interval $\left(t_{0}, t_{0}+\Delta t\right)$ to get [8]:

$$
\begin{aligned}
& \left.u\left(t_{0}+\Delta t\right)=u\left(t_{0}\right)+\Delta u_{t_{0}}^{\prime}+\Delta t, t_{0}\right), \\
& \Delta u\left(t, t_{0}\right) \equiv \int_{t_{0}}^{t} a\left(u\left(t^{\prime}\right)\right) d t^{\prime}+\int_{t_{0}}^{t} b\left(u\left(t^{\prime}\right)\right) d W\left(t^{\prime}\right) .
\end{aligned}
$$

One iteration of (3.2.2) in the calculation of $\Delta u\left(t_{0}+\Delta t, t_{0}\right)$ yields:

$$
\begin{aligned}
\Delta u\left(t_{0}+\Delta t, t_{0}\right) & =\int_{t_{0}}^{t_{0}+\Delta t} a\left(u\left(t_{0}\right)+\Delta u\left(t, t_{0}\right)\right) d t \\
& +\int_{t_{0}}^{t_{0}+\Delta t} b\left(u\left(t_{0}\right)+\Delta u\left(t, t_{0}\right)\right) d W(t),
\end{aligned}
$$

Next expand the integrands in (3.3) in a Taylor series around the value $u\left(t_{0}\right)$. For this purpose

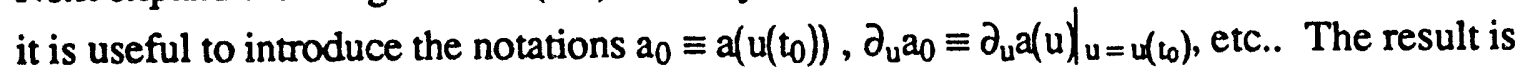

$$
\begin{aligned}
\Delta u\left(t_{0}+\Delta t, t_{0}\right)= & \Delta t a_{0}+b_{0} \int_{t_{0}}^{t_{0}+\Delta t} d W(t) \\
& +\partial_{u} a_{0} \int_{t_{0}}^{t_{0}+\Delta t} \Delta u\left(t, t_{0}\right) d t+\partial_{u} b_{0} \int_{t_{0}}^{t_{0}+\Delta t} \Delta u\left(t, t_{0}\right) d W(t) \\
& +\frac{1}{2} \partial_{u}^{2} a_{0}^{t_{0}+\Delta t}\left[\Delta u\left(t, t_{0}\right)\right]_{t_{0}}^{2} d t+\frac{1}{2} \partial_{u}^{2} b_{0} \int_{t_{0}}^{t_{0}+\Delta t}\left[\Delta u\left(t, t_{0}\right)\right]^{2} d W(t)+\cdots .
\end{aligned}
$$

Finally apply the Taylor series expansion to $\Delta u\left(t, t_{0}\right)$ in (3.2.2); this yields

$$
\Delta u\left(t, t_{0}\right)=\left(t-t_{0}\right) a_{0}+b_{0} W^{(1 / 2)}\left(t-t_{0}\right)+\cdots,
$$

where

$$
W^{(1 / 2)}\left(t-t_{0}\right) \equiv \int_{\infty}^{t} d W\left(t^{\prime}\right)=O\left(\Delta t^{1 / 2}\right)
$$


and the superscript $(1 / 2)$ has been added to the notation for the randc:n function $W$ as a reminder of the order-of-magnitude estimate $O\left(\Delta t^{1 / 2}\right)$, which follows from $t^{\prime}-t_{0} \leq \Delta t$ and the rule (2.7.2). When the series (3.5.1) is substituted into (3.4), the integrals on the right hand side are given by

$$
\begin{aligned}
& \int_{t_{0}}^{t_{0}+\Delta t} \Delta u\left(t, t_{0}\right) d t=\frac{1}{2} \Delta t^{2} a_{0}+W^{(3 / 2)}(\Delta t) b_{0}+\cdots \\
& W^{(3 / 2)}(\Delta t) \equiv \int_{t_{0}}^{t_{0}+\Delta t} W^{(1 / 2)}\left(t-t_{0}\right) d t=O\left(\Delta t^{3 / 2}\right), \\
& \int_{\varepsilon_{0}}^{t_{0}+\Delta t}\left[\Delta u\left(t, t_{0}\right)\right]^{2} d t=\frac{1}{3} \Delta t^{3} a_{0}^{2}+G^{(2)}(\Delta t) b_{0}^{2}+2 H^{(5 / 2)}(\Delta t) a_{0} b_{0}+\cdots, \\
& G^{(2)}(\Delta t) \equiv \int_{t_{0}}^{t_{0}+\Delta t}\left[W^{(1 / 2)}\left(t-t_{0}\right)\right]^{2} d t=O\left(\Delta t^{2}\right) \\
& H^{(5 / 2)}(\Delta t) \equiv \int_{t_{0}}^{t_{0}+\Delta t}\left(t-t_{0}\right) W^{(1 / 2)}\left(t-t_{0}\right) d t \\
& =\int_{t_{0}}^{t_{0}+\Delta t}\left(t-t_{0}\right) \frac{d}{d t} W^{(3 / 2)}\left(t-t_{0}\right) d t, \\
& =\Delta t \mathrm{~W}^{13 / 2}(\Delta \mathrm{t})-\mathrm{W}^{\mathrm{r} / 2 / 2}(\Delta \mathrm{t})=O\left(\Delta \mathrm{t}^{5 / 2}\right) \text {, } \\
& W^{(5 / 2)}(\Delta t)=\int_{t_{0}}^{t_{0}+\Delta t} W^{t^{3 / 2}}\left(t-t_{0}\right) d t=O\left(\Delta t^{5 / 2}\right) \\
& \int_{t_{0}}^{t_{0}+\Delta t} \Delta u\left(t, t_{0}\right) d W(t)=a_{0} I^{33 / 2}(\Delta t)+b_{0} C^{11}(\Delta t)+\cdots,
\end{aligned}
$$




$$
\begin{aligned}
& I^{(3 / 2)}(\Delta t) \equiv \int_{t_{0}}^{t_{0}+\Delta t}\left(t-t_{0}\right) d W(t) \\
& =\int_{t_{0}}^{t_{0}+\Delta t}\left\{d\left[\left(t-t_{0}\right) \bar{w}^{(1 / 2)}\left(t-t_{0}\right)\right]-W^{(1 / 2)}\left(t-t_{0}\right)\right\} \text {, } \\
& =\Delta t W^{(1 / 2)}(\Delta t)-W^{(3 / 2}(\Delta t)=O\left(\Delta t^{3 / 2}\right) \text {, } \\
& C^{1}(\Delta t) \equiv \int_{1_{0}}^{t_{0}+\Delta t} W^{(1 / 2)}\left(t-t_{0}\right) d W(t) \\
& =\frac{1}{2}\left(W^{(1 / 2)}(\Delta t)\right)^{2}-\frac{1}{2} \Delta t=O(\Delta t), \\
& \int_{t_{0}}^{t_{0}+\Delta t}\left[\Delta \mathrm{u}\left(\mathrm{t}, \mathrm{t}_{0}\right)\right]^{2} d W(\mathrm{t})=\mathrm{b}_{0}^{2} \mathrm{C}^{3 / 2)}(\Delta \mathrm{t})+2 \mathrm{a}_{0} \mathrm{~b}_{0} \mathrm{~K}^{(2)}(\Delta \mathrm{t})+\mathrm{a}_{0}^{2} \mathrm{~L}^{(5 / 2)}(\Delta \mathrm{t})+\cdots, \\
& C^{3 / 2}(\Delta t)=\int_{t_{0}}^{t_{0}+\Delta t}\left[W^{(1 / 2)}\left(t-t_{0}\right)\right]^{2} d W(t) \\
& =\frac{1}{3}\left[\mathrm{~W}^{(1 / 2)}(\Delta \mathrm{t})\right]^{3}-\mathrm{W}^{(3 / 2)}(\Delta \mathrm{t})=O\left(\Delta \mathrm{t}^{3 / 2}\right) \text {, } \\
& K^{(2)}(\Delta t)=\int_{t_{0}}^{t_{0}+\Delta t}\left(t-t_{0}\right) W^{(1 / 2)}\left(t-t_{0}\right) d W(t) \text {, } \\
& =\frac{1}{2} \int_{t_{0}}^{t_{0}+\Delta t}\left\{d\left[\left(t-t_{0}\right)\left(W^{(1 / 2)}\left(t-t_{0}\right)\right)^{2}\right]-\left(W^{(1 / 2)}\left(t-t_{0}\right)\right)^{2} d t\right\} \text {, } \\
& =\frac{1}{2} \Delta t\left(W^{(1 / 2)}(\Delta t)\right)^{2}-\frac{1}{2} G^{(2)}(\Delta t)=O\left(\Delta t^{2}\right) \text {, }
\end{aligned}
$$

12 


$$
\begin{aligned}
L^{(5 / 2)}(\Delta t) & =\int_{L_{0}}^{t_{0}+\Delta t}\left(t-t_{0}\right)^{2} d W(t), \\
& =\int_{t_{0}}^{t_{0}+\Delta t}\left\{d\left[\left(t-t_{0}\right)^{2} W^{(1 / 2)}\left(t-t_{0}\right)\right]-2\left(t-t_{0}\right) W^{(1 / 2)}\left(t-t_{0}\right) d t\right\}, \\
& =\Delta t^{2} W^{(1 / 2)}(\Delta t)-2 \Delta t W^{(3 / 2)}(\Delta t)+2 W^{(5 / 2)}(\Delta t)=O\left(\Delta t^{5 / 2}\right),
\end{aligned}
$$

where the rule (2.19) was used to obtain the final forms of (3.8.3) and (3.9.2).

Substituting these results into (3.2.1) yields, in the general (multiplicative) case, the formally exact series

$$
\begin{aligned}
u\left(t_{0}+\Delta t\right)= & u\left(t_{0}\right)+\Delta t a_{0}+\frac{1}{2} \Delta t^{2} a_{0} \partial_{u} a_{0}+\frac{1}{3} \Delta t^{3} a_{0}^{2} \partial_{u}^{2} a_{0} \\
& +W^{(1 / 2)} b_{0}+C^{(1)} b_{0} \partial_{u} b_{0}+\sum_{n=3}^{5} T^{(n / 2)}+\cdots, \\
T^{(3 / 2)}= & W^{(3 / 2)}\left(b_{0} \partial_{u} a_{0}-a_{0} \partial_{u} b_{0}\right)+\Delta t W^{(1 / 2)} a_{0} \partial_{u} b_{0}+C^{(3 / 2)} b_{0}^{2} \partial_{u}^{2} b_{0} \\
T^{(2)}= & G^{(2)} b_{0}\left(b_{0} \partial_{u}^{2} a_{0}-a_{0} \partial_{u}^{2} b_{0}\right) \\
& +\Delta t\left(W^{(1 / 2)}\right)^{2} a_{0} b_{0} \partial_{u}^{2} b_{0}, \\
T^{(5 / 2)}= & \Delta t^{2} W^{(1 / 2)} a_{0}^{2} \partial_{u}^{2} b_{0} \\
& +2\left(\Delta t W^{(3 / 2)}-W^{(5 / 2)}\right) a_{0}\left(b_{0} \partial_{u}^{2} a_{0}-a_{0} \partial_{u}^{2} b_{0}\right)
\end{aligned}
$$

where $W^{(1 / 2)}$ is understood to mean $W^{(1 / 2)}(\Delta t)$, etc. In the additive case, $\left(\partial_{\mathrm{u}} b=0\right)$, this result simplifies to

$$
\begin{gathered}
u\left(t_{0}+\Delta t\right)=u\left(t_{0}\right)+\Delta t a_{0}+\frac{1}{2} \Delta t^{2} a_{0} \partial_{u} a_{0}+\frac{1}{3} \Delta t^{3} a_{0}^{2} \partial_{u}^{2} a_{0} \\
+W^{(1 / 2)} b_{0}+\sum_{n=3}^{5} T^{(n / 2)}+\cdots, \\
T^{(3 / 2)}=W^{(3 / 2)} b_{0} \partial_{u} a_{0}, \\
T^{(2)}=G^{(2)} b_{0}^{2} \partial_{u}^{2} a_{0}, \\
T^{(5 / 2)}=2\left(\Delta t W^{(3 / 2)}-W^{(5 / 2)}\right) a_{0} b_{0} \partial_{u}^{2} a_{0},
\end{gathered}
$$




\section{$\underline{3 b} \quad$ Error estimates}

The formal series (3.10.1) appears to promise any desired accuracy if enough terms are kept. The accuracy actually obtainable in practice depends on the notion of convergence used. Two definitions are commonly employed: a strong notion based on the mean-square limit defined by (2.11), and a weaker notion defined with respect to the moments of the variable $u$. Let $u_{\Delta t}$ represent the formally exact solution obtained by summing the entire series in (3.10.1), and denote by $\tilde{\mathrm{u}}_{\Delta \mathrm{t}}$ an approximation to this series. Two kinds of approximations are involved: (1) truncation at a finite order, (2) approximate realizations of the fundamental Wiener process $\mathrm{dW}(\mathrm{t})$, and the other stochastic processes which enter into (3.10.1).

The approximation $\tilde{\mathrm{u}}_{\Delta \mathrm{t}}$ converges in the mean-square (norm) sense to $\mathrm{u}_{\Delta \mathrm{t}}$ if

$$
R_{m s}(\Delta t) \equiv\left\|u_{\Delta t}-\tilde{u}_{\Delta t}\right\| \equiv \sqrt{\left\langle\left(u_{\Delta t}-\tilde{u}_{\Delta t}\right)^{2}\right\rangle} \text { satisfies } \lim _{\Delta t \rightarrow 0} R_{m s}(\Delta t)=0
$$

and the approximation has an error $O\left(\Delta \mathrm{t}^{\mathrm{n}}\right)_{\mathrm{ms}}$ if

$$
\lim _{\Delta t \rightarrow 0} \frac{R_{m s}(\Delta t)}{\Delta t^{\alpha}}=0 \text { for all } \alpha<n
$$

This kind of convergence is importani when it is necessary to consider properties of the individual trajectories, e.g., in first passage time problems, distribution of extrema, or problems involving reflecting barriers. Note that many authors use $\left\langle\left(u_{\Delta t}-\tilde{u}_{\Delta t}\right)^{2}\right\rangle$, rather than the norm defined above, to measure errors [8]; the term norm used in the definition is intended as a reminder of this fact.

The approximation $\tilde{\mathrm{u}}_{\Delta \mathrm{t}}$ converges moment-wise to $\mathrm{u}_{\Delta \mathrm{t}}$ if

$$
R_{\text {mom }}^{(m)}(\Delta t) \equiv\left|\left\langle\left(u_{\Delta t}\right)^{m}\right\rangle-\left\langle\left(\tilde{u}_{\Delta t}\right)^{m}\right\rangle\right| \text { satisfies } \lim _{\Delta t \rightarrow 0} R_{m o m}^{(m)}(\Delta t)=0
$$

for all $m \geq 0$, and the approximation has an error of $O\left(\Delta t^{n}\right)_{\text {mom }}$ if

$$
\lim _{\Delta t \rightarrow 0} \frac{R_{m o m}^{(m)}(\Delta t)}{(\Delta t)^{\alpha}}=0 \text { for all } \alpha<n
$$

The definition of moment-wise convergence is most easily understood by rewriting (3.10.1) as 


$$
u\left(t_{0}+\Delta t\right)=D_{\Delta t}+S_{\Delta t}
$$

where $D_{\Delta t}$ and $S_{\Delta t}$ are respectively called the deterministic and stochastic parts:

$$
\begin{aligned}
& D_{\Delta t} \equiv u\left(t_{0}\right)+\Delta t a_{0}+\frac{1}{2} \Delta t^{2} a_{0} \partial_{u} a_{0}+\frac{1}{3} \Delta t^{3} a_{0}^{2} \partial_{u}^{2} a_{0}+\cdots \\
& S_{\Delta t} \equiv W^{(1 / 2)} b_{0}+C^{(1)} b_{0} \partial_{u} b_{0}+\sum_{n=3}^{5} T^{(n / 2)}+\cdots
\end{aligned}
$$

The reason for this terminology is that $\mathrm{u}\left(\mathrm{t}_{0}\right)$ is either the initial value, or the result of a previous step; therefore in any given realization of the solution, $u\left(t_{0}\right)$ and $a_{0}$ have definite values. Thus in calculating moments of $u\left(t_{0}+\Delta t\right)$, we can assume that $D_{\Delta t}$ does not fluctuate, i.e., $\left\langle D_{\Delta t}\right\rangle=D_{\Delta t}$. Then the $m$-th moment of the formal solution can be expressed as

$$
\begin{aligned}
\left\langle\left(u_{\Delta t}\right)^{m}\right\rangle= & \left\langle\left(D_{\Delta t}+S_{\Delta t}\right)^{m}\right\rangle \\
& =\left(D_{\Delta t}\right)^{m}+m\left(D_{\Delta t}\right)^{m-1}\left\langle S_{\Delta t}\right\rangle+\left(\begin{array}{c}
m \\
2
\end{array}\right)\left(D_{\Delta t}\right)^{m-2}\left\langle\left(S_{\Delta t}\right)^{2}\right\rangle+\ldots
\end{aligned}
$$

The same formula holds for the approximate solution $\tilde{\mathrm{u}}_{\Delta t}$ if $D_{\Delta t}$ and $S_{\Delta t}$ are replaced by the approximate forms, i.e., $D_{\Delta t} \rightarrow \widetilde{D}_{\Delta t}$ and $S_{\Delta t} \rightarrow \widetilde{S}_{\Delta t}$. Thus the moment-wise error estimator (for the $\mathrm{m}$-th moment) is

$$
\begin{aligned}
& R_{m o m}^{(m)}(\Delta t)=\left|\delta_{\Delta t}^{(m)}\right| \\
& \mathcal{E}_{\Delta t}^{(m)}=\left(D_{\Delta t}\right)^{m}-\left(\widetilde{D}_{\Delta t}\right)^{m}+m\left\langle\left(D_{\Delta t}\right)^{m-1}\left\langle s_{\Delta t}\right\rangle-\left(\widetilde{D}_{\Delta t}\right)^{m-1}\left\langle\widetilde{s}_{\Delta t}\right)\right\}+ \\
& \quad\left(\begin{array}{c}
m \\
2
\end{array}\right)\left\{\left(D_{\Delta t}\right)^{m-2}\left\langle S_{\Delta t}^{2}\right\rangle-\left(\widetilde{D}_{\Delta t}\right)^{m-2}\left\langle\widetilde{s}_{\Delta t}^{2}\right)\right\}+\cdots
\end{aligned}
$$

To show how these notions work in practice, I first apply the idea of mean-square convergence to the differencing scheme

$$
\tilde{u}\left(t_{0}+\Delta t\right)=u\left(t_{0}\right)+\Delta t a_{0}+W^{(1 / 2)} b_{0}+C^{(1)} b_{0} \partial_{u} b_{0}+T^{(3 / 2)}
$$

where $T^{(3 / 2)}$ is given by (3.10.2). Since we are interested in the error produced in a single step, the initial values for the exact and approximate schemes are the same, $\widetilde{u}\left(t_{0}\right)=u\left(t_{0}\right)$. The quantities $W^{(n / 2)}(\Delta t)$ in (3.10.2) are linear functionals of the basic Wiener process $d W(t)$ and are therefore readily simulated. The quantities $C^{(n / 2)}(\Delta t)$ are nonlinear functionals of $d W(t)$, but they can be evaluated in terms of $W^{(n / 2)}(\Delta t)$, according to (3.8.3) and (3.9.2). Comparing (3.17) to (3.10.1), we see that 


$$
\begin{aligned}
& \mathrm{u}_{\Delta \mathrm{t}}-\tilde{\mathrm{u}}_{\Delta \mathrm{t}}=\frac{1}{2} \Delta \mathrm{t}^{2} \mathrm{a}_{0} \partial_{\mathrm{u}} \mathrm{a}_{0}+\mathrm{T}^{(2)}+O\left(\Delta \mathrm{t}^{5 / 2}\right) \\
& \mathrm{R}_{\mathrm{ms}}(\Delta \mathrm{t})=O\left(\Delta \mathrm{t}^{2}\right)_{\mathrm{ms}}
\end{aligned}
$$

Thus the scheme (3.17) is accurate, in the mean-square sense, through $O\left(\Delta \mathrm{t}^{3 / 2}\right)_{\mathrm{ms}}$. The result (3.18.1) also shows the difficulty met when attempting to improve the single step accuracy. According to (3.10.3), $T^{(2)}$ depends on the nonlinear functional $G^{(2)}$ which, unlike $C^{(n / 2)}$, cannot be evaluated in closed form. There does not seem to be any simple, computationally practical method to simulate $G^{(2)}$; therefore it is impractical to obtain single step accuracies of higher order than $\left(\Delta t^{3 / 2}\right)$ ms. Since the coefficient of $G^{(2)}$ in (3.10.3) does not vanish when $\partial_{u} b_{0}=0$, this problem applies to additive as well as multiplicative noise. The corresponding scheme for additive noise is

$$
\tilde{u}\left(t_{0}+\Delta t\right)=u\left(t_{0}\right)+W^{(1 / 2)} b_{0}+\Delta t a_{0}+W^{(3 / 2)} b_{0} \partial_{u} a_{0}
$$

which has the same error estimate. Thus for the generic case, the best way to improve the ms-accuracy of the solution is to use successively smaller values of $\Delta t$ and extrapolate the results to $\Delta t=0$. The special class of problems in which the Wronskian [17] $\mathrm{W}[\mathrm{a}, \mathrm{b}] \equiv \mathrm{a}(\mathrm{u}) \partial_{\mathrm{u}} \mathrm{b}(\mathrm{u})-\mathrm{b}(\mathrm{u}) \partial_{\mathrm{u}} \mathrm{a}(\mathrm{u})$ does not depend on $\mathrm{u}$ would allow improvement up to errors of $\left(\Delta t^{5 / 2}\right)_{m s}$. In this case the coefficient of $G^{(2)}$ in (3.10.3) vanishes and $T^{(2)}$ can be simulated.

Next consider the same difference scheme using moment-wise error estimators. The approximate deterministic and stochastic parts are

$$
\begin{aligned}
& \widetilde{D}_{\Delta t} \equiv u\left(t_{0}\right)+\Delta t a_{0} \\
& \tilde{S}_{\Delta t}=W^{(1 / 2)} b_{0}+C^{(1)} b_{0} \partial_{u} b_{0}+T^{(3 / 2)}
\end{aligned}
$$

Since the deterministic part has errors $O\left(\Delta \mathrm{t}^{2}\right)$, the moment-wise error estimate is $O\left(\Delta \mathrm{t}^{2}\right)_{\text {mom }}$, the same as the mean square estimate. The important difference between the two notions of convergence is that it is possible to improve the single-step error for moment-wise error estimates. As an example, consider the improved scheme

$$
\begin{aligned}
& \widetilde{D}_{\Delta t} \equiv u\left(t_{0}\right)+\Delta t a_{0}+\frac{1}{2} \Delta t^{2} a_{0} \partial_{u} a_{0}, \\
& \widetilde{S}_{\Delta t} \equiv W^{(1 / 2)} b_{0}+C^{(1)} b_{0} \partial_{u} b_{0}+T^{(3 / 2)}+T^{(2)},
\end{aligned}
$$


which gives an error estimate $O\left(\Delta \mathrm{t}^{5 / 2}\right)$ mom, at the cost of including the $\mathrm{T}^{(2)}$ term which prevented the same improvement in the mean square case. This difficulty is avoided liere, since only the moments of the solution are required. Note that $\tilde{S}_{\Delta t}=O\left(\Delta t^{1 / 2}\right)$ so that the approximate moments are given by

$$
\begin{aligned}
\left\langle\left(\tilde{\mathrm{u}}_{\Delta t}\right)^{\mathrm{m}}\right\rangle= & \left(\widetilde{D}_{\Delta t}\right)^{\mathrm{m}}+\mathrm{m}\left(\widetilde{D}_{\Delta t}\right)^{\mathrm{m}-1}\left\langle\widetilde{S}_{\Delta t}\right\rangle+\left(\begin{array}{c}
m \\
2
\end{array}\right)\left(\widetilde{D}_{\Delta t}\right)^{m-2}\left\langle\left(\tilde{S}_{\Delta t}\right)^{2}\right\rangle \\
& +\left(\begin{array}{c}
m \\
3
\end{array}\right)\left(\widetilde{D}_{\Delta t}\right)^{m-3}\left\langle\left(\widetilde{S}_{\Delta t}\right)^{3}\right\rangle+\left(\begin{array}{c}
m \\
3
\end{array}\right)\left(\widetilde{D}_{\Delta t}\right)^{m-4}\left\langle\left(\tilde{S}_{\Delta t}\right)^{4}\right\rangle+O\left(\Delta t^{5 / 2}\right) .
\end{aligned}
$$

This result shows that only the first four moments of the stochastic part are required for the evaluation of an arbitrary moment of the solution with accuracy $O\left(\Delta t^{2}\right)$ mom. In evaluating these moments one can use the identities $\left\langle\mathrm{W}^{(\mathrm{t} / 2)}\right\rangle=0$, which follow from the observation that $W^{(n / 2)}$ is a linear functional of dW. The nonlinear functionals $C^{n / 2)}$ also satisfy $\left\langle C^{n / 2)}\right\rangle=0$ by virtue of the fact that $\mathrm{W}^{(1 / 2)}$ is a Gaussian process normalized by (2.7.2). Combining these facts with the definition (3.21.2) yields

$$
\begin{aligned}
& \begin{aligned}
\begin{aligned}
\left.\tilde{S}_{\Delta t}\right\rangle
\end{aligned}=\left\langle T^{(2)}\right\rangle, \\
\begin{aligned}
\left\langle\left(\tilde{S}_{\Delta t}\right)^{2}\right\rangle & =\left\langle\left(W^{(1 / 2)}\right)^{2}\right\rangle b_{0}^{2}+2 b_{0}^{2} \partial_{u} b_{0}\left\langle W^{(1 / 2)} C^{(1)}\right\rangle+2 b_{0}\left\langle W^{(1 / 2)} T^{(3 / 2)}\right\rangle \\
& +\left\langle\left(C^{(1)}\right)^{2}\right\rangle\left(b_{0} \partial_{u} b_{0}\right)^{2}+O\left(\Delta t^{5 / 2}\right)
\end{aligned}
\end{aligned} \\
& \begin{aligned}
\left\langle\left(\tilde{S}_{\Delta t}\right)^{3}\right\rangle & =3\left\langle\left(W^{(1 / 2)}\right)^{2} C^{(1)}\right\rangle b_{0}^{3} \partial_{u} b_{0}+O\left(\Delta t^{5 / 2}\right)
\end{aligned} \\
& \left\langle\left(\tilde{S}_{\Delta t}\right)^{4}\right\rangle=\left\langle\left(W^{(1 / 2)}\right)^{4}\right\rangle b_{0}^{4}+O\left(\Delta t^{5 / 2}\right)
\end{aligned}
$$

Thus an improvement of the error estimate to $O\left(\Delta \mathrm{t}^{5 / 2}\right)_{\text {mom }}$ only requires the evaluation of the first moment of $\mathrm{T}^{(2)}$, rather than a full simulation. The first moment is given by, $c f(3.10 .3)$,

$$
\begin{aligned}
\left\langle T^{(2)}\right\rangle= & \left\langle G^{(2)}\right\rangle b_{0}\left(b_{0} \partial_{u}^{2} a_{0}-a_{0} \partial_{u}^{2} b_{0}\right) \\
+ & \Delta t\left\langle\left(W^{(1 / 2)}\right)^{2}\right\rangle a_{0} b_{0} \partial_{u}^{2} b_{0}, \\
= & \left\langle G^{(2)}\right\rangle b_{0}\left(b_{0} \partial_{u}^{2} a_{0}-a_{0} \partial_{u}^{2} b_{0}\right) \\
& +\Delta t^{2} a_{0} b_{0} \partial_{u}^{2} b_{0},
\end{aligned}
$$

where the final form was obtained by using (2.7.2). The definition (3.7.2) of $\mathrm{G}^{(2)}$ yields, after another use of (2.7.2), 


$$
\begin{aligned}
\left\langle G^{(2)}\right\rangle & =\int_{t_{0}}^{t_{0}+\Delta t}\left\langle\left[W^{(1 / 2)}\left(t-t_{0}\right)\right]^{2}\right\rangle d t, \\
& =\int_{t_{0}}^{t_{0}+\Delta t}\left(t-t_{0}\right) d t, \\
& =\frac{1}{2} \Delta t^{2} .
\end{aligned}
$$

The other moments can be calculated in a similar way. To begin the evaluation of (3.23.2) note that (3.8.3) implies $\left\langle W^{(1 / 2)} C^{(1)}\right\rangle=0$, and that (3.10.2) gives

$$
\begin{gathered}
\left\langle W^{(1 / 2)} T^{(3 / 2)}\right\rangle=\left\langle W^{(1 / 2)} W^{(3 / 2)}\right\rangle\left(b_{0} \partial_{u} a_{0}-a_{0} \partial_{u} b_{0}\right)+\Delta t\left\langle\left(W^{(1 / 2)}\right)^{2}\right\rangle a_{0} \partial_{u} b_{0} \\
+\left\langle W^{(1 / 2)} C^{(3 / 2)}\right\rangle b_{0}^{2} \partial_{u}^{2} b_{0}
\end{gathered}
$$

The averages on the right hand side can be evaluated by judicious use of the interval rules (2.7), but it is generally more convenient to use the formally equivalent rule

$$
\left\langle d W\left(t_{1}\right) d W\left(t_{2}\right)\right\rangle=\delta\left(t_{1}-t_{2}\right) d t_{1} d t_{2}
$$

Combining (3.27) with (3.5.2), (3.6.2), (3.9.2), and (3.8.3) yields

$$
\begin{aligned}
\left\langle\left(W^{(1 / 2)}(\Delta t)\right)^{2}\right\rangle & =\int_{t_{0}}^{t_{0}+\Delta t} \int_{t_{0}}^{t_{0}+\Delta t}\left\langle d W\left(t_{1}\right) d W\left(t_{2}\right)\right\rangle \\
& =\int_{t_{0}}^{t_{0}+\Delta t} \int_{t_{0}}^{t_{0}+\Delta t} \delta\left(t_{1}-t_{2}\right) d t_{1} d t_{2} \\
& =\Delta t, \\
\left\langle W^{(1 / 2)} W^{(3 / 2)}\right\rangle & =\int_{t_{0}}^{t_{0}+\Delta t} d t\left\langle W^{(1 / 2)}(\Delta t) W^{(1 / 2)}\left(t-t_{0}\right)\right\rangle, \\
& =\int_{t_{0}}^{t_{0}+\Delta t} d t \int_{t_{0}}^{t_{0}+\Delta t} \int_{t_{0}}^{t}\left\langle d W\left(t_{1}\right) d W\left(t_{2}\right)\right\rangle, \\
& =\frac{1}{2} \Delta t^{2}, \\
\left\langle W^{(1 / 2)} C^{(3 / 2)}\right\rangle & =\frac{1}{3}\left\langle\left(W^{(1 / 2)}\right)^{4}\right\rangle-\left\langle W^{(1 / 2)} W^{(3 / 2)}\right\rangle, \\
& =\frac{1}{3} 3 \Delta t^{2}-\frac{1}{2} \Delta t^{2}=\frac{1}{2} \Delta t^{2},
\end{aligned}
$$




$$
\begin{aligned}
& \left\langle\left(W^{(1 / 2)}\right)^{2} C^{(1)}\right\rangle=\frac{1}{2}\left\langle\left(W^{(1 / 2)}\right)^{4}\right\rangle-\frac{1}{2}\left\langle\left(W^{(1 / 2)}\right)^{2}\right\rangle \Delta t=\Delta t^{2}, \\
& \left\langle\left(W^{(1 / 2)}\right)^{4}\right\rangle=3 \Delta t^{2} \\
& \left\langle\left(C^{(1)}\right)^{2}\right\rangle=\frac{1}{4}\left\langle\left(W^{(1 / 2)}\right)^{4}\right\rangle+\frac{1}{4} \Delta t^{2}-\frac{1}{2} \Delta t\left\langle\left(W^{(1 / 2)}\right)^{2}\right\rangle=\frac{1}{2} \Delta t^{2}
\end{aligned}
$$

The necessary moments are then given by

$$
\begin{aligned}
& \left\langle\tilde{S}_{\Delta t}\right\rangle=\frac{1}{2} b_{0}\left(b_{0} \partial_{u}^{2} a_{0}+a_{0} \partial_{u}^{2} b_{0}\right) \Delta t^{2} \\
& \left\langle\left(\tilde{S}_{\Delta t}\right)^{2}\right\rangle=\Delta t b_{0}^{2}+\Delta t^{2} b_{0}\left\{b_{0} \partial_{u} a_{0}+a_{0} \partial_{u} b_{0}+b_{0}^{2} \partial_{u}^{2} b_{0}+\frac{1}{2} b_{0}\left(\partial_{u} b_{0}\right)^{2}\right\} \\
& \left\langle\left(\tilde{S}_{\Delta t}\right)^{3}\right\rangle=3 \Delta t^{2} b_{0}^{3} \partial_{u} b_{0} \\
& \left\langle\left(\tilde{S}_{\Delta t}\right)^{4}\right\rangle=3 \Delta t^{2} b_{0}^{4}
\end{aligned}
$$

Further increases in single-step accuracy demand the evaluation of higher moments of nonlinear functionals like $\mathrm{G}^{(2)}$, and the labor rapidly becomes prohibitive.

\section{3c Systems of autonomous Langevin equations}

The autonomous form of the system of equations (2.28) is

$$
d u^{\lambda}=a^{\lambda}(u) d t+b^{\lambda \mu}(u) d W_{\mu}(t),
$$

and the corresponding finite-difference form is

$$
\begin{aligned}
& u^{\lambda}\left(t_{0}+\Delta t\right)=u^{\lambda}\left(t_{0}\right)+\Delta t a_{0}^{\lambda}+\frac{1}{2} \Delta t^{2} a_{0}^{\mu} \partial_{\mu} a_{0}^{\lambda}+W_{\mu}^{(1 / 2)} b_{0}^{\lambda \mu}+C_{\rho \sigma}^{(1)} b_{0}^{\rho \mu} \partial_{\mu} b_{0}^{\lambda \sigma} \\
& +\sum_{n=3}^{4} T^{\lambda(n / 2)}+\cdots \\
& T^{\lambda(3 / 2)}=W_{\sigma}^{(3 / 2)}\left(b_{0}^{\mu \sigma} \partial_{\mu} a_{0}^{\lambda}-a_{0}^{\mu} \partial_{\mu} b_{0}^{\lambda \sigma}\right)+\Delta t W_{\sigma}^{(1 / 2)} a_{0}^{\mu} \partial_{\mu} b_{0}^{\lambda \sigma} \\
& +\frac{1}{2} C_{\sigma \rho \beta}^{(3 / 2)} b_{0}^{\mu \rho} b_{0}^{\alpha \beta} \partial_{\mu} \partial_{\alpha} b_{0}^{\lambda \sigma} \text {, }
\end{aligned}
$$




$$
\begin{aligned}
& T^{\lambda(2)}=\frac{1}{2}\left(\partial_{\mu} \partial_{\alpha} a_{0}^{\lambda}\right) b_{0}^{\mu \rho} b_{0}^{\alpha \sigma} G_{\rho \sigma}^{(2)}, \\
& W_{\lambda}^{(1 / 2)}\left(t-t_{0}\right) \equiv \int_{t_{0}}^{t} d W_{\lambda}\left(t^{\prime}\right), \\
& C_{\alpha \beta}^{(1)} \equiv \int_{t_{0}}^{t_{0}+\Delta t} d W_{\alpha} W_{\beta}^{(1 / 2)}\left(t-t_{0}\right), \\
& W_{\lambda}^{(3 / 2)}(\Delta t) \equiv \int_{t_{0}}^{t_{0}+\Delta t} W_{\lambda}^{(1 / 2)}\left(t-t_{0}\right) d t, \\
& C_{\lambda \mu v}^{(3 / 2)} \equiv \int_{t_{0}}^{t_{0}+\Delta t} d W_{\lambda} W_{\mu}^{(1 / 2)}\left(t-t_{0}\right) W_{v}^{(1 / 2)}\left(t-t_{0}\right), \\
& G_{\alpha \beta}^{(2)} \equiv \int_{t_{0}}^{t_{0}+\Delta t} d t W_{\alpha}^{(1 / 2)}\left(t-t_{0}\right) W_{\beta}^{(1 / 2)}\left(t-t_{0}\right) .
\end{aligned}
$$

The definitions of mean square (norm) convergence and moment-wise convergence are taken over from the scalar case by applying them separately to each component $u^{\lambda}$. The vector quantities $W_{\lambda}^{(n / 2)}$ create no difficulties since they are linear functionals of the independent Wiener processes $d W_{\lambda}$, but the first order tensor $C_{\alpha \beta}^{(1)}$ is a nonlinear functional and, in contrast to the scalar case, it does pose a problem. The diagonal components $C_{\alpha \alpha}^{(1)}$ can be evaluated by (3.8.3), but there is no simple way to simulate the off-diagonal components, $C_{\alpha \beta}^{(1)}$ for $\alpha \neq \beta$. Thus for vector equations with multiplicative noise the highest order practical scheme for mean square (norm) convergence is

$$
\tilde{u}^{\lambda}\left(t_{0}+\Delta t\right)=u^{\lambda}\left(t_{0}\right)+W_{\mu}^{(1 / 2)} b_{0}^{\lambda \mu}
$$

with error $O(\Delta \mathrm{t})_{\mathrm{ms}}$, while in the additive case one can use

$$
\tilde{u}^{\lambda}\left(t_{0}+\Delta t\right)=u^{\lambda}\left(t_{0}\right)+\Delta t a_{0}^{\lambda}+W_{\mu}^{(1 / 2)} b_{0}^{\lambda \mu}+W_{\mu}^{(3 / 2)} b_{0}^{\sigma \mu} \partial_{\sigma} a_{0}^{\lambda}
$$

with error $O\left(\Delta \mathrm{t}^{2}\right)_{\mathrm{ms}}$.

Just as in the scalar case, it is possible to achieve higher single-step accuracy in the 
moment-wise sense by evaluating a small number of moments of the nonlinear functionals which are difficult to simulate. In the vector case the difficulties occur, at a lower order, e.g., $C_{\alpha \beta}^{(1)}$, so the attainable accuracy will still be lower than in the scalar case.

\section{Simulation of the Wiener process}

Comparing the definition (2.7.4) of $\Delta \mathrm{W}$ with (3.5.2) for $\mathrm{W}^{(1 / 2)}$ yields

$$
W^{(1 / 2)}(\Delta t)=\Delta W\left(t_{0}\right)
$$

i.e., (2.7) shows that the quantities $W^{(1 / 2)}(\Delta t)$ are Gaussian random variables with mean zero and variance $\Delta t$, to be chosen independently for successive time steps. Thus if we set $W^{(1 / 2)}(\Delta t)=\sqrt{\Delta t} \xi$, the problem is to generate Gaussian random niumbers with zero mean and unit variance. There are many ways to do this exactly [18], but I will describe below only the so called Polar (Box-Muller) algorithm [19] which is the most commonly used method. In addition, I will describe an approximate method[20] which depends on the central limit theorem. [15] For a system of $n$ Langevin equations, the scalar procedure must be repeated $n$ times with independent random numbers.

\section{4a The Polar Method}

This method produces a pair of independent Gaussian random variables by the following algorithm:

(1) Choose two independent numbers uniformly distributed in $[0,1]$ and map them into $[-1,1]$

$\mathrm{U}_{1}=\operatorname{Ranf}\left(\right.$ ), $\mathrm{U}_{2}=\operatorname{Ranf}(), \mathrm{V}_{1}=2 \mathrm{U}_{1}-1, \mathrm{~V}_{2}=2 \mathrm{U}_{2}-1$

(2) Reject all points $\left(\mathrm{V}_{1}, \mathrm{~V}_{2}\right)$ outside the unit circle $S=V_{1}^{2}+V_{2}^{2}$, If $[S>1]$ go to $(1)$

(3) Define $\xi_{1}$ and $\xi_{2}$ by

$$
\xi_{j}=V_{j} \sqrt{\frac{-2 \ln S}{S}}, j=1,2
$$

Then $\xi_{1}$ and $\xi_{2}$ are independent Gaussian random numbers with zero mean and unit variance.

4b A central limit approximation 
The Polar method is in principle exact, but it involves a substantial amount of calculation to produce the pairs of Gaussian variables. In many applications, it is not necessary to produce exactly Gaussian distributed random numbers. In particular, when only a few moments of the soiution are required, it is sufficient to choose the $\xi$ 's so that the first two moments are correct. One way of doing that is to use the central limit theorem in the following algorithm [20]

(1) $\eta_{j}=\operatorname{Ranf}(), j=1, \cdots, M$

(2) $\xi=\sqrt{\frac{12}{M}} \sum_{j=1}^{M}\left(\eta_{j}-\frac{1}{2}\right)$

Choose $\mathrm{M}$ random numbers uniformly distributed in $[0,1]$ and form the normalized sum according to (4.3). The first two moments agree with the Gaussian distribution for any $M$ :

$$
\langle\xi\rangle=0,\left\langle\xi^{2}\right\rangle=1
$$

but the higher moments do not correctly factorize except in the limit $M \rightarrow \infty$, for which the central limit theorem guarantees that $\xi$ will obey a Gaussian distribution. In practice a moderate value such as $M=10$ will usually suffice.

\section{4c Linear functionals of the Wiener process}

The linear functionals required for these calculations can all be expressed as

$$
f d W] \equiv \int_{t_{0}}^{t_{0}+\Delta t} f(t) d W(t)
$$

All quantities of this form are Gaussian random variables satisfying

$$
\langle f[d W] g[d W]\rangle=\int_{t_{0}}^{t_{0}+\Delta t} f(t) g\left(t^{\prime}\right) \int_{L_{0}}^{t_{0}+\Delta t}\left\langle d W(t) d W\left(t^{\prime}\right)\right\rangle=\int_{L_{0}}^{t_{0}+\Delta t} f(t) g(t)
$$

which, for $f=g$ specializes to the normalization condition

$$
\left\langle(f[d W])^{2}\right\rangle=\int_{t_{0}}^{t_{0}+\Delta t}|f(t)|^{2}
$$


These results follow immediately from the formal rule (3.27). The general rule (4.6.1) shows that two linear functionals $\mathrm{f}[\mathrm{dW}]$ and $g[\mathrm{dW}]$ are statistically independent if and only if the corresponding density functions $f$ and $g$ are orthogonal on the interval $\left[t_{0}, t_{0}+\Delta t\right]$. In the general case it will be necessary to simulate $\mathrm{N}$ such functionals defined by density functions $f_{1}, \cdots, f_{N}$ which are not necessarily orthogonal. The general procedure is to first normalize the functions by

$$
\bar{f}_{k} \equiv \frac{f_{k}}{\left\|f_{k}\right\|},\left\|f_{k}\right\| \equiv\left[\int_{t_{0}}^{t_{0}+\Delta t}\left|f_{k}(t)\right|^{2}\right.
$$

and then to define the real symmetric correlation matrix

$$
K_{i j} \equiv\left\langle\bar{f}_{i} \bar{f}_{j}\right\rangle
$$

with real eigenvalues $\lambda_{(m)}$ and a complete orthonormal set of eigenvectors $F_{(m)}$, $\mathrm{m}=1, \mathrm{~m}=1, \cdots, \mathrm{N}$. Then it is easy to see that the variables $\xi_{(\mathrm{m})}$ defined by

$$
\xi_{(m)}=\frac{\sum_{j=1}^{N} F_{(m) j} \bar{f}_{j}}{\sqrt{\lambda_{(m)}}}
$$

satisfy $\left\langle\xi_{(m)} \xi_{(n)}\right\rangle=\delta_{m n}$, i.e., they are a set of independent Gaussian random variables. The equations (4.9) can be inverted by using the completeness relation

$$
\sum_{m=1}^{N} F_{(m) k} F_{(m) j}=\delta_{k j}
$$

to derive

$$
\bar{f}_{j}[d W]=\sum_{j=1}^{N} \sqrt{\lambda_{(m)}} F_{(m) j} \xi_{(m)}
$$

Thus the linear functionals $f_{j}$ can be simulated by generating $N$ independent Gaussian random variables $\xi_{(1)}, \cdots, \xi_{(\mathrm{N})}$ and defining 


$$
f_{j}[d W]=\left\|f_{j}\right\| \sum_{j=1}^{N} \sqrt{\lambda_{(m)}} F_{(m) j} \xi_{(m)}
$$

As an example consider the additive-noise difference scheme (3.19). In this case $f_{1}[d W]=W^{(1 / 2)}$ and $f_{2}[d W]=W^{(3 / 2)}$ where (3.5.2) and (3.8.2) yield $f_{1}(t)=1$ and $f_{2}(t)=t_{0}+\Delta t-t$ respectively. The $2 \times 2$ correlation matrix is then

$$
K=\left[\begin{array}{cc}
1 & \sqrt{3} / 2 \\
\sqrt{3} / 2 & 1
\end{array}\right]
$$

and the general result (4.11) becomes

$$
\begin{aligned}
& W^{(1 / 2)}=\sqrt{\frac{\Delta t}{2}}\left\{\sqrt{\lambda_{(1)}} \xi_{1}+\sqrt{\lambda_{(2)}} \xi_{2}\right\}, \\
& W^{(3 / 2)}=\frac{(\Delta t)^{3 / 2}}{\sqrt{6}}\left\{\sqrt{\lambda_{(1)}} \xi_{1}-\sqrt{\lambda_{(2)}} \xi_{2}\right\}, \\
& i_{41)}=!+\frac{\sqrt{3}}{2}, \lambda_{(2)}=1-\frac{\sqrt{3}}{2}
\end{aligned}
$$

Thus the correlated processes $W^{(1 / 2)}$ and $W^{(3 / 2)}$ are constructed from the independent processes $\xi_{1}$ and $\xi_{2}$ which could, for example, be generated by the polar method.

\section{Stochastic partial differential equations}

Stochastic partial differential equations arise in many contexts, e.g.: reaction-diffusion problems with fluctuating sources[1], propagation of electromagnetic fields in media with random optical properties[7], simulations of quantum field theory[21], fluid turbulence [22], the theory of amplified spontaneous emission [23], etc. The mathematical theory has been greatly strengthened in recent years, but the numerical simulation methods ars still not as well developed as those based on Ito calculus. In order to have a concrete subjec'. for discussion, I will consider the generalized heat equation

$$
\partial_{t} u(r, t)=\frac{1}{2} K \nabla^{2} u(r, t)+B(r, u) \eta(r, t)
$$

where $\operatorname{Re}(K) \geq 0, \nabla^{2}$ is the Laplace operator, and $\eta$ is a fluctuating quantity which satisfies

$$
\langle\eta(\mathbf{r}, \mathfrak{t})\rangle=0
$$




$$
\left\langle\eta(\mathbf{r}, \mathbf{t}) \eta\left(\mathbf{r}^{\prime}, \mathrm{t}^{\prime}\right)\right\rangle=\delta\left(\mathbf{r}-\mathbf{r}^{\prime}\right) \delta\left(\mathrm{t}-\mathrm{t}^{\prime}\right)
$$

The form of $\mathrm{B}(\mathbf{r}, \mathrm{u})$ varies according to the application involved. When $\mathrm{K}$ is real and $B(r, t, u) \rightarrow B(r),(5.1)$ can be interpreted as an equation for the local temperature of a fluid with a spatially inhomogeneous, fluctuating heat source which represents additive noise. [1] When $\mathrm{K}$ is purely imaginary and $\mathrm{B}(\mathbf{r}, \mathrm{t}, \mathrm{u}) \rightarrow \mathrm{B}(\mathbf{r}) \mathrm{u}(\mathbf{r}, \mathrm{t}),(5.1)$ describes paraxial propagation of an electromagnetic wave in a medium with a randomly fluctuating index of refraction. [7], [24]

In the following subsections I will show that the singular conditions (5.2) can be replaced by mathematically meaningful statements employing the Wiener process, and then discuss a less rigorous approach which forms the basis for most practical simulations.

\section{5a Infinite dimensional Langevin equations}

The formal equation (5.1) can be interpreted as an infinite system of Langevin equations by making use of the fact that the solutions of the underlying deterministic problem are supposed to lie in some definite space of functions. For the sake of simplifying the explanation, I will assume that the space in question is a (real) Hilbert space with inner product

$$
(\phi, \psi)=\int d^{3} r \phi(r) \psi(\mathbf{r}),
$$

but the results generalize readily to complex Hilbert spaces or to more general spaces. First rewrite (5.1) as

$$
d u(r, t)=\frac{1}{2} K \nabla^{2} u(r, t) d t+B(r, u) \eta(r, t) d t
$$

and introduce a basis for the Hilbert space $\left\{\phi_{1}, \phi_{2}, \ldots\right\}$ satisfying

$$
\begin{aligned}
& \left(\phi_{\lambda}, \phi_{\mu}\right)=\delta_{\lambda \mu} \text { (orthonormality) } \\
& \sum \phi_{\lambda}(\mathbf{r}) \phi_{\lambda}\left(\mathbf{r}^{\prime}\right)=\delta\left(\mathbf{r}-\mathbf{r}^{\prime}\right) \text { (completeness) }
\end{aligned}
$$

Next define $u^{\lambda}(t)=\left(\phi_{\lambda}(\cdot), u(\cdot, t)\right)$ and take the inner product of (5.4) with $\phi_{\lambda}$ to obtain

$$
d u^{\lambda}(t)=a^{\lambda}(u)+\sum_{\mu} b^{\lambda \mu}(u) d W_{\mu}(t)
$$




$$
\begin{aligned}
& \mathrm{a}^{\lambda}(\mathrm{u})=\frac{1}{2} \mathrm{~K} \sum_{\beta}\left(\phi_{\lambda}, \nabla^{2} \phi_{\beta}\right) \mathrm{u}^{\beta}(\mathrm{t}), \\
& \mathrm{b}^{\lambda \mu}(\mathrm{u})=\left(\phi_{\lambda}(\cdot), \mathrm{B}(\cdot, \mathrm{u}) \phi_{\mu}(\cdot)\right), \\
& d W_{\lambda}(\mathrm{t})=\left(\phi_{\lambda}(\cdot), \eta(\cdot, \mathrm{t})\right) \mathrm{dt} .
\end{aligned}
$$

The path to mathematical respectability is now clear. We use the completeness relation (5.5.2) to solve (5.6.4) for $\eta$ :

$$
\eta(\mathbf{r}, \mathrm{t}) \mathrm{dt}=\sum_{\lambda} \phi_{\lambda}(\mathbf{r}) \mathrm{d} \mathrm{W}_{\lambda}(\mathrm{t})
$$

Next interpret $\left\{d W_{\lambda}(t)\right\}$ as a family of independent Wiener processes and use (5.7) as the definition of $\eta$. The rigorous rule (2.29) for a family of independent Wiener processes can be formally restated as

$$
\left\langle d W_{\lambda}\left(t_{1}\right) d W_{\mu}\left(t_{2}\right)\right\rangle=\delta \lambda_{\mu} \delta\left(t_{1}-t_{2}\right) d t_{1} d t_{2}
$$

which simplifies the following calculation:

$$
\begin{aligned}
\left\langle\eta(\mathbf{r}, \mathrm{t}) \eta\left(\mathbf{r}^{\prime}, \mathrm{t}^{\prime}\right)\right\rangle \mathrm{dt} d \mathrm{t}^{\prime} & =\sum_{\lambda} \sum_{\mu} \phi_{\lambda}(\mathbf{r}) \phi_{\mu}\left(\mathbf{r}^{\prime}\right)\left\langle d W_{\lambda}(\mathrm{t}) d \mathrm{~W}_{\mu}\left(\mathrm{t}^{\prime}\right)\right\rangle \\
& =\sum_{\lambda} \sum_{\mu} \phi_{\lambda}(\mathbf{r}) \phi_{\mu}\left(\mathbf{r}^{\prime}\right) \delta_{\lambda \mu} \delta\left(t-t^{\prime}\right) d t d t^{\prime} \\
& =\delta\left(\mathbf{r}-\mathbf{r}^{\prime}\right) \delta\left(t-t^{\prime}\right) d t d t^{\prime}
\end{aligned}
$$

where the completeness condition (5.5.2) was used to get the last line. Thus the singular rules (5.2) can be replaced by the mathematically satisfactory properties of the independent Wiener processes.

The interpretation (5.6) of the stochastic partial differential equation (5.1) suggests that one simulation technique would be to truncate the infinite set of equations (5.6.1) by keeping some finite number of basis functions. The problem could then be treated as outlined in Sec. 3c.

The success of this approach depends on a judicious choice of the basis $\left\{\phi_{1}, \phi_{2}, \ldots\right\}$ so that the most important aspects of the problem can be captured by a relatively small number of basis elements. Since this choice is often not at all obvious, most simulations make use of a discretization approach discussed in the next section. 


\section{5b Discretization}

Discretization is in effect another way to replace the partial differential equation by a finite, though large, set of ordinary differential equations. The idea is to treat the stochastic equation (5.1) as though it were a deterministic equation and then to make special provision for the noise term. The first step is to consider a rectangular lattice with spacing $\ell$, so that the spatial grid points are located at $\mathbf{R}_{\mathbf{j}}=\ell\left(\mathrm{j}_{\mathrm{x}} \hat{\mathbf{x}}+\mathrm{j}_{\mathrm{y}} \hat{\mathbf{y}}+\mathrm{j}_{\mathrm{z}} \hat{\mathbf{z}}\right)$, where $\mathrm{j}_{\mathrm{x}}, \mathrm{j}_{\mathrm{y}}$, and $\mathrm{j}_{\mathrm{z}}$ are integers. Next define an averaging function $f(\mathbf{r})$ which vanishes outside a cube of side $\ell$ centered on the origin and is normalized by

$$
\int d^{3} r f(r)=1
$$

The value of $u(r, t)$ near $\mathbf{R}_{\mathbf{j}}$ will be represented by its average

$$
u J(t) \equiv \int d^{3} r f_{j}(r) u(r, t), f_{j}(r) \equiv f\left(r-R_{j}\right)
$$

The equations satisfied by the $u J(t)$ can be obtained from (5.6.1) by multiplying through by $\left(\mathrm{f}_{\mathrm{j}}, \phi_{\lambda}\right)$ and summing over $\lambda$ Thus

$$
\begin{aligned}
& \sum_{\lambda}\left(f_{j}, \phi_{\lambda}\right) d u^{\lambda}=d u l \\
& \sum_{\lambda}\left(f_{j}, \phi_{\lambda}\right) a^{\lambda}(u)=\frac{1}{2} K\left(f_{j}, \nabla^{2} u\right), \\
& \sum_{\lambda}\left(f_{j}, \phi_{\lambda}\right) b^{\lambda \mu}(u)=\left(f_{j}, B(\cdot, u) \phi_{\mu}(\cdot)\right) .
\end{aligned}
$$

The averaging function can be chosen so that

$$
\left(f_{j}, \nabla^{2} u\right)=\sum_{k} D_{j k} u^{k}+O(\ell)
$$

where the coefficients $D_{j k}$ define a finite difference approximation to $\nabla^{2}$. The most important approximation is to replace the function $B(r, u)$ on the right hand side of $(5.12 .3)$ by its approximate average value at $\mathbf{R}_{\mathbf{j}}, B\left(\mathbf{R}_{\mathbf{j}}, u^{j}(t)\right)$. With these approximations the $u^{\mathrm{J}}(\mathrm{t})$ 's satisfy 


$$
\begin{aligned}
& \left.d u^{j}(t)=\frac{1}{2} K \sum_{k} D_{j k} u^{k}(t)+B\left(R_{j}, u^{j}(t)\right) d W_{j}(t) \quad \text { (no sum on } \mathbf{j}\right), \\
& d W_{j}(t) \equiv \sum_{\mu}\left(f_{j}, \phi_{\mu}\right) d W_{\mu}(t) .
\end{aligned}
$$

The relations (5.8), together with the completeness conditions (5.5) give

$$
\left\langle d W_{j}(t) d W_{k}\left(t^{\prime}\right)\right\rangle=\left(f_{j}, f_{k}\right) \delta\left(t-t^{\prime}\right) d t d t^{\prime}
$$

The definition of the averaging function shows that $\left(f_{j}, f_{k}\right) \equiv \delta_{j k}$, so the Wiener processes $\mathrm{W}_{\mathrm{j}}(\mathrm{t})$ at each grid point can be treated as independent. Thus an independent Wiener process must be generated at each spatial grid point, and the resulting Langevin equations (5.14.1) must be solved by one of the methods outlined in Sec. $3 \mathrm{c}$.

\section{An example from optical switching theory}

The derivation of the system of Langevin equations corresponding to a given physical problem is not always completely straightforward. An interesting example of the difficulties that can arise in practice is provided by the use of optical bistability in all-optical switching.[25] Certain materials, e.g., InSb, possess a strongly nonlinear dielectric susceptibility which can lead to an abrupt change in the transmission through the sample when the pump laser reaches a critical (switching) intensity $I_{s}$. Thus the sample will be effectively opaque for $\mathrm{I}<\mathrm{I}_{\mathrm{s}}$ and essentially transparent for $\mathrm{I}>\mathrm{I}_{\mathrm{s}}$. This is called optical bistability. When using this phenomenon to produce a switch, it is desirable to operate the pump laser as close to the switching value as possible. On the other hand, if the intensity is too close to $I_{s}$ the inevitable random fluctuations in laser frequency and intensity could cause spontaneous switching. In order to guard against this behavior, it is necessary to estimate the mean time before spontaneous switching as a function of $\left(I-I_{s}\right) I_{0}$, where $I_{0}$ is a scale intensity. It turns out that the fluctuations in frequency, $\delta \omega(t)=\omega(t)-\omega_{0}$, are the most important, so the intensity fluctuations will be neglected. The frequency noise has a small amplitude, $\left|\delta \omega(t) / \omega_{0}\right|<1$, and fluctuates rapidly, i.e., the correlation time $\tau_{\mathrm{f}}$ is short compared to any deterministic response time for the nonlinear material.

Maxwell's equations lead to an equation for the phase shift $\phi_{n l}$ due to propagation through the nonlinear material. The object of interest is the phase deviation $u=\phi_{n l}-\phi_{0}+t_{R} \delta \omega$, where $\phi_{0}$ is the steady state phase change in the absence of noise and $t_{R}$ is the round trip time in the optical cavity. For small phase deviations $u$ and small fluctuations $f \equiv \delta \omega / \omega_{0}$, one finds 


$$
\begin{aligned}
& \frac{d u}{d t}=-\frac{u}{T_{0}}+A_{2} u^{2}+\Gamma \Phi_{0} g, \\
& g=f+\frac{1}{\Gamma} \frac{d f}{d t}
\end{aligned}
$$

where $T_{0}, \Gamma$, and $A_{2}$ are constants characterizing the deterministic response of the nonlinear medium and $\Phi_{0}=\phi_{0}+t_{R} \omega_{0}$. The correlation time for the frequency noise satisfies $\tau_{\mathrm{f}}<\mathrm{T}_{0}$ and $\Gamma \tau_{f} \ll 1$. The conditions on $f$ are usually satisfied by modelling it as a white noise source, i.e., by setting $f d t=\sqrt{\kappa_{1}} d W(t)$, where $\kappa$ characterizes the strength of the fluctuations and $\mathrm{dW}(\mathrm{t})$ is a Wiener process. Formally this becomes

$$
\left\langle f\left(t_{1}\right) f\left(t_{2}\right)\right\rangle=\kappa_{1} \delta\left(t_{1}-t_{2}\right)
$$

To see how the white noise model works in the application at hand, consider the correlation function for the noise term $\mathrm{g}$ :

$$
\begin{aligned}
& \left\langle g\left(t_{1}\right) g\left(t_{2}\right)\right\rangle=\left\langle\left(f\left(t_{1}\right)+\frac{1}{\Gamma} \frac{d f\left(t_{1}\right)}{d t_{1}}\right)\left(f\left(t_{2}\right)+\frac{1}{\Gamma} \frac{d f\left(t_{2}\right)}{d t_{2}}\right)\right\rangle, \\
& =\left\langle f\left(t_{1}\right) f\left(t_{2}\right)\right\rangle+\frac{1}{\Gamma}\left\langle f\left(t_{1}\right) \frac{d f\left(t_{2}\right)}{d t_{2}}\right\rangle+\frac{1}{\Gamma}\left\langle f\left(t_{2}\right) \frac{d f\left(t_{1}\right)}{d t_{1}}\right\rangle \\
& +\frac{1}{\Gamma^{2}}\left\langle\frac{\mathrm{df}\left(\mathrm{t}_{2}\right)}{\mathrm{dt}_{2}} \frac{\mathrm{df}\left(\mathrm{t}_{1}\right)}{\mathrm{dt}_{1}}\right\rangle
\end{aligned}
$$

The identity

$$
\left\langle f\left(t_{1}\right) \frac{d f\left(t_{2}\right)}{d t_{2}}\right\rangle=\frac{\partial}{\partial t_{2}}\left\langle f\left(t_{1}\right) f\left(t_{2}\right)\right\rangle=-\frac{\partial}{\partial t_{1}}\left\langle f\left(t_{1}\right) f\left(t_{2}\right)\right\rangle=-\left\langle f\left(t_{2}\right) \frac{d f\left(t_{1}\right)}{d t_{1}}\right\rangle,
$$

which is valid for a stationary process, simplifies (6.3) to

$$
\begin{aligned}
\left\langle g\left(t_{1}\right) g\left(t_{2}\right)\right\rangle & =\left\langle f\left(t_{1}\right) f\left(t_{2}\right)\right\rangle+\frac{1}{\Gamma^{2}}\left\langle\frac{d f\left(t_{2}\right)}{d t_{2}} \frac{d f\left(t_{1}\right)}{d t_{1}}\right\rangle, \\
& =\left\langle f\left(t_{1}\right) f\left(t_{2}\right)\right\rangle-\frac{1}{\Gamma^{2}} \frac{\partial}{\partial t_{1}^{2}}\left\langle f\left(t_{1}\right) f\left(t_{2}\right)\right\rangle
\end{aligned}
$$

The power spectrum $P_{g}(v)$ for $g$ is then

$$
\begin{aligned}
P_{g}(v) & =\int d\left(t_{1}-t_{2}\right) e^{i v\left(t_{1}-t_{2}\right)}\left\langle g\left(t_{1}\right) g\left(t_{2}\right)\right\rangle, \\
& =\kappa_{1}\left(1+\frac{v^{2}}{\Gamma^{2}}\right)
\end{aligned}
$$


This is much too noisy, since the power spectrum actually diverges as the frequency $v \rightarrow \infty$, consequently the customary white noise model for $f$ is not physically acceptable in this case. As an altemative model one might consider assuming $g$ itself to be proportional to a Wiener process, i.e., $g(t)=\sqrt{\kappa_{2}} \eta(t)$, where $\eta$ satisfies (2.2). Then (6.1.2) would become a definition for $f$ :

$$
\frac{d f}{d t}=-\Gamma f+\sqrt{k_{2}} \Gamma \eta(t)
$$

A random process defined by an equation of the form (6.7) is called an Ornstein-Uhlenbeck process, and the explicit solution of (6.7),

$$
f(t)=\sqrt{\kappa_{2}} \Gamma \int_{-\infty}^{t} d t^{\prime} e^{-\Gamma\left(t-t^{\prime}\right)} \eta\left(t^{\prime}\right)
$$

yields the correlation function

$$
\left\langle f\left(t_{1}\right) f\left(t_{2}\right)\right\rangle=\frac{\kappa_{2} \Gamma}{2} e^{-\Gamma \mid t_{1}-t_{2}}
$$

Thus the assumption that $\mathrm{g}$ itself is a white noise source implies that the correlation time for the frequency fluctuations $\mathrm{f}$ is $\tau_{\mathrm{f}}=1 / \Gamma$. This violates the condition $\Gamma \tau_{\mathrm{f}} \ll 1$ which states that the frequency fluctuations are rapid on the scale of the response rates of the material; consequently this choice must also be rejected. Retreating one step further, let us assume that $f$ is an Ornstein-Uhlenbeck process with correlation time $\tau_{\mathrm{f}}$ satisfying $\Gamma \tau_{\mathrm{f}} \ll<$,

$$
\begin{aligned}
& \frac{d f}{d t}=-\frac{1}{\tau_{f}} f+\sqrt{\kappa_{3}} \eta(t), \\
& \left\langle f\left(t_{1}\right) f\left(t_{2}\right)\right\rangle=\frac{\kappa_{3} \tau_{f}}{2} e^{-\mid t_{1}-t / \tau_{f}}, \\
& P_{f}(v)=\frac{\kappa_{3} \tau_{f}^{2}}{1+v^{2} \tau_{f}^{2}} .
\end{aligned}
$$

With this model for $f$, the power spectrum for $g$ is

$$
P_{g}(v)=\left(1+\frac{v^{2}}{\Gamma^{2}}\right) P_{f}(v)=\kappa_{3} \tau_{f}^{2} \frac{1+\frac{v^{2}}{\Gamma^{2}}}{1+v^{2} \tau_{f}^{2}},
$$


which is asymptotically no worse than white noise. Thus the original problem, which involved only one system variable $u(t)$, has been replaced by the two-dimensional system

$$
\begin{aligned}
& d u^{\lambda}=a^{\lambda}(u, t) d t+b^{\lambda \mu}(u, t) d W_{\mu}(t),(\lambda, \mu=1,2), \\
& \mathrm{u}^{1}=\mathrm{u}-\Phi_{0} \mathrm{f}, \\
& \mathrm{u}^{2}=\mathrm{f}, \\
& a(u)=\left(\begin{array}{c}
-\frac{1}{T_{0}}\left(u^{1}+\Phi_{0} u^{2}\right)+A_{2}\left(u^{1}+\Phi_{0} u^{2}\right)^{2}+\Gamma \Phi_{0} u^{2} \\
-\frac{1}{\tau_{f}} u^{2}
\end{array}\right) \\
& \mathrm{b}(\mathrm{u})=\left(\begin{array}{cc}
0 & 0 \\
0 & \sqrt{\mathrm{K}_{3}}
\end{array}\right)
\end{aligned}
$$

In this particular problem, only one Wiener process is required, since $\mathrm{dW}_{1}$ is missing from the equations. Since the question here involves escape or first passage time, the relevant convergence criterion is the mean-square (norm) notion discussed in Sec.3b. A rough answer which is suitable for most purposes can be obtained from the corresponding Fokker-Planck equation [25] which leads to a standard Arrhenius formula [1]

This work was performed under the auspices of the U.S. Department of Energy by Lawrence Livermore National Laboratory under contract No. W-7405-ENG-48.

\section{References}

1. Gardiner, C.W., Handbook of Stochastic Methods. 2nd ed. Springer Series in Synergetics, ed. H. Haken. Vol. 13. 1985, Berlin: Springer-Verlag.

2. Van Kampen, N.G., Phys. Lett. C, 1976. 24(3): p. 171-228.

3. Arnold, L., Stochastic Differential Equations: Theory and Applications. 1974, New York: Wiley.

4. Bharucha-Reid, A.T., Elements of the Theory of Markov Processes and their Applications. 1960, New York: McGraw-Hill. 
5. Gihman, I.I. and A.V. Skorohod, The Theory of Stochastic Proceses. Vol. I,II,III. 1974, Berlin: Springer-Verlag.

6. Stratonovich, R.L., Topics in the Theory of Random Noise. Vol. I,II. 1963, .

7. Blankenship, G.L. and J.S. Baras, SIAM J. Appl. Math., 1981. 41(3): p. 518-552.

8. Greiner, A., W. Strittmatter, and J. Honerkamp, J. Stat. Phys., 1988. 51(1): p. 95-108.

9. Milshtein, G.N., Theory Prob. Appl., 1978. 23: p. 396-401.

10. Rao, N.J., J.D. Borwankar, and D. Ramakrishna, SIAM j. Control, 1974. 12(1): p. 124139.

11. Rümelin, W., SIAM J. Numer. Anal., 1982. 19(3): p. 604-613.

12. Einstein, A., Ann. Phys. (Leipzig), 1905. 17: p. 549.

13. Smoluchowski, M.v., Ann. Phys. (Leipzig), 1906. 21: p. 756.

14. Langevin, P., C. R. Acad. Sci., 1908. 146: p. 530.

15. Feller, W., An Introduction to Probability Theory and Its Applications. 2nd ed. 1957, New York: JohnWiley \& Sons.

16. Riesz, F.a.S.-N., B., Functional Analysis. 2nd ed. 1955, New York: Frederick Ungar Publishing Co.

17. Morse, P.M.a.F., H., Methods of Theoretical Physics. 1fst ed. International Series in Pure and Applied Physics, ed. L.I. Schiff. Vol. 1. 1953, New York: McGraw-Hill Booke Co., Inc.

18. Knuth, D.E., The Art of Computer Programming. 2nd ed. Addison-Wesley eries in Computer Science and Information Processing, ed. M.A. Harrison. 1981, Reading, Mass.: Addison-Wesley.

19. Box, G.E.P., Muller, M. E., and Marsaglia, G., Ann. Math. Stat., 1958. 28: p. 610.

20. Risken, H., The Fokker-Planck Equation. 2nd ed. Springer Series in Synergetics, ed. H. Haken . Vol. 18. 1989, Berlin: Springer-Verlag. 
21. Borkar, V.S.a.M., S. K. Lattice approximation in the stochastic quantization of $\left(\phi^{4}\right)_{2}$ fields. in Stochastic Differential Equations and Applications II. 1988. Trento, Italy: SpringerVerlag.

22. Monin, A.S.a.Y., A. M., Statistical Fluid Mechanics, Mechanics of Turbulence. 1971, Cambridge, Mass.: MIT Press.

23. Garrison, J.C., Ritchie, B.,Nathel, H.,Hong, C. K.,and Minner, L., Phys. Rev. A, 1991. 43: p. 4941-4953.

24. Leland, R.P., Stochastic models for laser propagation in atmospheric turbulence. Lecture Notes in Control and Information Scilences, Vol. 133. 1989, New York: Springer-Verlag.

25. Filipowicz, P., Garrison, J. C., Meystre, P., and Wright , E. M., Phys. Rev. A, 1987. 35: p. 1172-1180. 

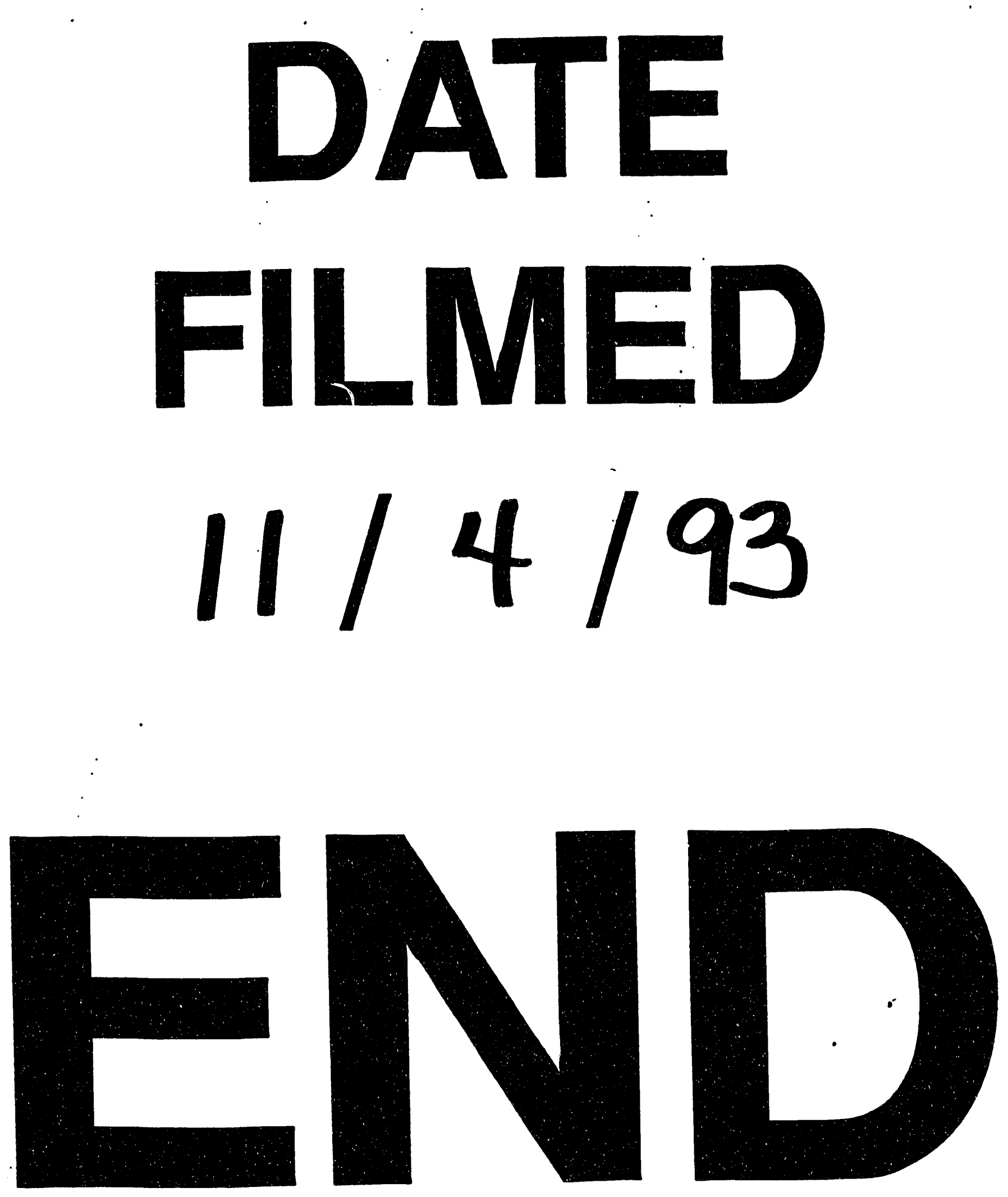
- 